\title{
The persistent homology of a sampled map: from a viewpoint of quiver representations
}

\author{
Hiroshi Takeuchi ${ }^{1}$
}

Received: 11 December 2019 / Accepted: 5 January 2021 / Published online: 4 March 2021

(c) The Author(s) 2021

\begin{abstract}
This paper is intended to introduce a filtration analysis of sampled maps based on persistent homology, providing a new method for reconstructing the underlying maps. The key idea is to extend the definition of homology induced maps of correspondences using the framework of quiver representations. Our definition of homology induced maps is given by most persistent direct summands of representations. The direct summands uniquely determine a persistent homology. We provide stability theorems of this process and show that the output persistent homology of the sampled map is the same as that of the underlying map if the sample is sufficiently dense. Compared to existing methods using eigenspace functors, our filtration analysis represents an important advantage that no prior information related to the eigenvalues of the underlying map is required. Some numerical examples are given to demonstrate the effectiveness of our method.
\end{abstract}

Keywords Computational topology · Persistent homology · Quiver representations · Reconstruction of maps

Mathematics Subject Classification 55N35 $\cdot$ 16G20 $\cdot$ 37B99 $\cdot$ 18A99 $\cdot 55$ U99

\footnotetext{
I would like to thank Yasuaki Hiraoka for introducing me to this topic, and for many fruitful discussions and stimulating questions. I also thank Emerson G. Escolar for important questions about the well-definedness of the persistence analysis. I also extend my appreciation to Zin Arai for his constructive suggestions and comments on the paper. I acknowledge support by Japan Society for the Promotion of Science KAKENHI Grant No. 16J03138, Japan Society for the Promotion of Science KAKENHI Grant No. 19KK0068, and Japan Science and Technology Agency CREST Mathematics 15656429.

$凶$ Hiroshi Takeuchi

hiroshi-takeuchi@biwako.shiga-u.ac.jp

1 Shiga University, 1-1-1 Banba, Hikone, Shiga 522-8522, Japan
} 
Fig. 1 Graph $\operatorname{Gr}(f)$ of $f$ and the graph of the sampled map

Fig. 2 Both spaces are divided into grids and elicit a correspondence $F$, which approximates the graph $\operatorname{Gr}(f)$
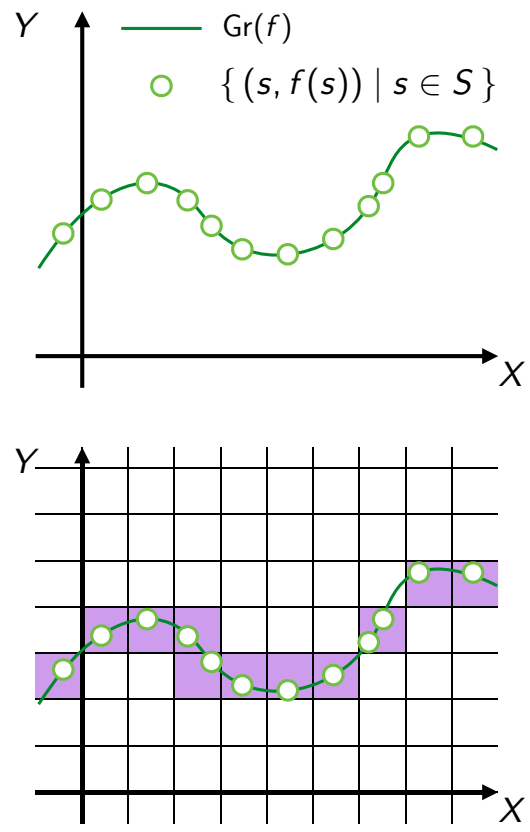

\section{Introduction}

One can consider the following problem.

Problem 1.1 Let $X$ and $Y$ be topological spaces, and let $f: X \rightarrow Y$ be a continuous map. If we know only $X, Y$, and sampling data $f \uparrow_{S}$, which is a restriction of $f$ on a finite subset $S \subset X$, then can we retrieve any information about the homology induced map $f_{*}: H X \rightarrow H Y$ ?

The map $f \uparrow_{S}$ is called a sampled map of $f$. This paper is motivated by Harker et al. (2016), who suggested the following analysis for sampled maps (Fig. 1). A grid $\mathcal{X}$ of $X$ is a finite collection of subsets of $X$ with disjoint interiors such that $\bigcup \mathcal{X}:=\bigcup_{X^{\prime} \in \mathcal{X}} X^{\prime}=X$. First, we divide the topological spaces $X$ and $Y$ into grids $\mathcal{X}$ and $\mathcal{Y}$ and let $F$ be the union of regions with elements of the sample $\operatorname{Gr}\left(f \uparrow_{S}\right):=$ $\{(s, f(s)) \mid s \in S\}$. That is,

$$
F:=\left\{(x, y) \in X \times Y \mid x \in \exists X^{\prime} \in \mathcal{X}, y \in \exists Y^{\prime} \in \mathcal{Y},\left(X^{\prime} \times Y^{\prime}\right) \cap \operatorname{Gr}\left(f \uparrow_{S}\right) \neq \emptyset\right\},
$$

the purple regions in Fig. 2. The set $F$ is an approximation of the graph $\operatorname{Gr}(f)$ from the sampled map by this subspace, which is called a correspondence.

Definition 1.2 A correspondence $F$ from $X$ to $Y$ is a subspace of $X \times Y$.

Definition 1.3 For a correspondence $F$, let $p: F \rightarrow X$ and $q: F \rightarrow Y$ be canonical projections, and let $p_{*}: H F \rightarrow H X$ and $q_{*}: H F \rightarrow H Y$ be their homology induced maps. If $p_{*}$ and $q_{*}$ satisfy two properties 
- $\operatorname{Im} p_{*}=H X$ (homologically complete)

- $q_{*}\left(\operatorname{Ker} p_{*}\right)=0$ (homologically consistent),

then the induced map of $F$ defined as $F_{*}:=q_{*} \circ p_{*}^{-1}: H X \rightarrow H Y$ is well-defined.

The graph $\operatorname{Gr}(f)$ of $f$ is a correspondence. Because $f$ is continuous, $p: \operatorname{Gr}(f) \rightarrow$ $X$ is a homeomorphism. As a consequence, $p_{*}$ and $q_{*}$ for $\operatorname{Gr}(f)$ satisfy homological completeness and homological consistency. Therefore $\operatorname{Gr}(f)_{*}$ is well-defined. We remark that this induced map $\operatorname{Gr}(f)_{*}$ coincides with $f_{*}$. The following theorem guarantees that $F_{*}$ restores $f_{*}$ when the grid is fine and the sample is sufficiently dense.

Theorem 1.4 (Harker et al. 2016, Theorem 3.10) If a correspondence $F$ satisfies $\operatorname{Gr}(f) \subset F$ and is homologically consistent, then $F_{*}$ is well-defined and $f_{*}=F_{*}$.

In Sect. 3, we give a new definition of induced maps of correspondences within the quiver representations framework. It might be readily apparent that the indecomposable decompositions of quiver representations give us an assignment among the bases of $H X, H F$, and $H Y$, which defines the induced map from $H X$ to $H Y$.

Edelsbrunner et al. (2015) reported a means of analyzing the eigenspaces of the homology induced map of a self-map (discrete dynamical system). For this analysis, the authors construct a filtration of simplicial maps from the sampled map and build its persistent homology by application of the homology functor and eigenspace functors. This construction of the filtration and the new definition of homology induced maps provide a further technique that enables another persistence analysis of sampled maps, shown in Sect. 4. Specifically, the bases' assignment can compress the three persistent homologies generated by the finite sets $S, f \uparrow_{S}$, and $f(S)$, yielding a persistent homology that describes the persistence of the topological mapping from the domain to the image of the induced map $f_{*}$. Moreover, in Sect. 4.2, we apply this persistence analysis to the above gridded setting.

The main theorems of this paper are stability theorems for these processes, Theorems 5.6 and 5.8 in Sect. 5, which state that these mappings from the input (sampled maps) to output (persistent homology or persistence diagrams) are non-expanding maps. The key idea for the proof is functoriality of our analysis and is applied to 2-D persistence modules in Sect. 6.

Finally, we present some numerical results in Sect. 7 and examples of failed reconstructions in Sect. 8.

\section{Preliminaries}

In this section, we introduce quiver representations and matrix notation for morphisms between $A_{n}$ type representations. For more details, we refer the reader respectively to Assem et al. (2006) and Asashiba et al. (2019).

\subsection{Quivers and their representations}

Throughout this paper, scalars of vector spaces and coefficient rings of homology groups are a fixed field $K$. A quiver $Q=\left(Q_{0}, Q_{1}, s, t\right)$ (or simply $\left(Q_{0}, Q_{1}\right)$ ) is a 
directed graph with a set of vertices $Q_{0}$, a set of arrows $Q_{1}$, and morphisms $s, t: Q_{1} \rightarrow$ $Q_{0}$ identifying the source and the target vertex of an arrow. An arrow $\alpha \in Q_{1}$ is denoted by $\alpha: s(\alpha) \rightarrow t(\alpha)$. A representation of a quiver $Q$, denoted $M=\left(M_{a}, \varphi_{\alpha}\right)_{a \in Q_{0}, \alpha \in Q_{1}}$ (or simply $\left(M_{a}, \varphi_{\alpha}\right)$ or $(M, \varphi)$ ), is a collection of a (finite-dimensional) vector space $M_{a}$ for each vertex $a \in Q_{0}$ and a linear map $\varphi_{\alpha}: M_{a} \rightarrow M_{b}$ for each arrow $\alpha: a \rightarrow$ $b \in Q_{1}$.

A morphism from $M=\left(M_{a}, \varphi_{\alpha}\right)$ to $M^{\prime}=\left(M_{a}^{\prime}, \varphi_{\alpha}^{\prime}\right)$ is defined as

$$
f:=\left\{f_{a}: M_{a} \rightarrow M_{a}^{\prime} \mid a \in Q_{0}\right\}: M \rightarrow M^{\prime}
$$

with commutativity

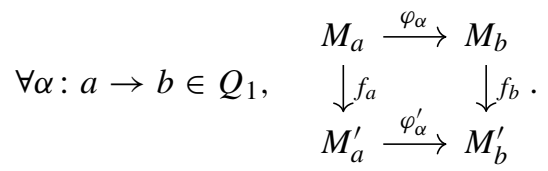

The composition of morphisms $f=\left\{f_{a}\right\}: M \rightarrow M^{\prime}$ and $g=\left\{g_{a}\right\}: M^{\prime} \rightarrow M^{\prime \prime}$ is $g f=\left\{g_{a} f_{a}\right\}: M \rightarrow M^{\prime \prime}$.

These definitions determine an additive category of representations rep $(Q)$. Specifically, $\operatorname{rep}(Q)$ has a zero representation, isomorphisms of representations, and direct sums of representations. One can see the concrete construction of these in Assem et al. (2006).

A representation $M$ is indecomposable if $M \cong N \oplus N^{\prime}$ implies $N=0$ or $N^{\prime}=0$. From the Krull-Remak-Schmidt theorem, every representation $M$ can be uniquely decomposed into a direct sum of indecomposables $M \cong N_{1} \oplus \cdots \oplus N_{s}$, unique up to isomorphism and permutations.

A quiver $Q$ is of finite type if the number of distinct isomorphism classes of indecomposables is finite. It is of infinite type otherwise.

$A_{n}\left(\tau_{n}\right)$ type quivers (or simply $A_{n}$ type quivers) are a class of quivers with the following shape:

$$
A_{n}\left(\tau_{n}\right): \stackrel{1}{\circ} \longleftrightarrow \stackrel{2}{\circ} \longleftrightarrow \cdots \longleftrightarrow \begin{aligned}
& n \\
& \circ
\end{aligned}
$$

where $\longleftrightarrow$ denotes a forward arrow $\longrightarrow$ or backward arrow $\longleftarrow$, and where $\tau_{n}$ is a sequence of $n-1$ symbols $f$ and $b$ determining the arrow orientation. From Gabriel's theorem (Gabriel 1972), every $A_{n}$ type representation

$$
M: M_{1} \longleftrightarrow M_{2} \longleftrightarrow \cdots \longleftrightarrow M_{n}
$$

can be decomposed uniquely into a direct sum of indecomposable interval representations as

$$
M \cong \bigoplus_{1 \leq b \leq d \leq n} \mathbb{I}[b, d]^{m_{b, d}} \quad\left(m_{b, d} \in \mathbb{Z}_{\geq 0} \text { : multiplicity }\right)
$$




$$
\mathbb{I}[b, d]: 0 \longleftrightarrow \cdots \longleftrightarrow 0 \longleftrightarrow \stackrel{b-\text { th }}{\longleftrightarrow} \stackrel{\mathrm{id}_{K}}{\longleftrightarrow} K \stackrel{\mathrm{id}_{K}}{\longleftrightarrow} \cdots \stackrel{\mathrm{id}_{K}}{\longleftrightarrow} \stackrel{d \text {-th }}{\longleftrightarrow} \longleftrightarrow 0 \longleftrightarrow \cdots \longleftrightarrow 0
$$

In topological data analysis, persistent homology plays a central role. The homology of a filtration of simplicial complexes

$$
H X: H X_{1} \rightarrow H X_{2} \rightarrow \cdots \rightarrow H X_{n}
$$

can be regarded as a representation of an $A_{n}(f f \cdots f)$ type quiver in the framework of quiver representations. Each interval representation $\mathbb{I}[b, d]$ corresponds to a generator of a homology group that is born at $H X_{b}$ and which dies at $H X_{d+1}$. Therefore, the length $d-b$ is called its lifetime or persistence. The persistence diagram is a multiset

$$
D_{M}=\left\{(b, d) \mid 1 \leq b \leq d \leq n,(b, d) \text { has multiplicity } m_{b, d}\right\},
$$

determined by the decomposition. We illustrate it by plotting it on a plane. This description provides an overview of the generators of all homology groups. Therefore, this approach is used frequently for applications of persistent homology.

The framework of quiver representations has extended persistent homology to general representations of quivers. We designate representations of quivers as persistence modules. Zigzag persistence modules (Carlsson and de Silva 2010) are examples of the extension, enabling persistence analysis of time series data.

Consider deformations of topological spaces $\left(X_{1}, \ldots, X_{T}\right)$, a sequence of topological spaces. The zigzag persistence of the sequence is the representation of an $A_{2 T-1}(f b f b \cdots f b)$ type quiver of

$$
H\left(X_{1}\right) \rightarrow H\left(X_{1} \cup X_{2}\right) \leftarrow H\left(X_{2}\right) \rightarrow \cdots \rightarrow H\left(X_{T-1} \cup X_{T}\right) \leftarrow H X_{T}
$$

composed by the unions of neighboring spaces and their canonical inclusions. Decomposition of the zigzag persistence module as a representation yields a persistence diagram again, where each interval captures the persistence of a homology generator in the deformations of spaces.

\subsection{Matrix notation for morphisms in $\operatorname{rep}\left(A_{n}\right)$}

Asashiba et al. (2019) established a new matrix notation for morphisms in $\operatorname{rep}\left(A_{n}\right)$ in the following way. This notation will provide a clear perspective when arguing the well-definedness of persistence analysis in Sect. 4.

Definition 2.1 (Asashiba et al. 2019, Definition 3) The arrow category $\operatorname{arr}(\operatorname{rep}(Q))$ of the category $\operatorname{rep}(Q)$ is a category with objects that are all morphisms in $\operatorname{rep}(Q)$, where morphisms are defined as explained below. For two objects $f: M \rightarrow N$ and $f^{\prime}: M^{\prime} \rightarrow N^{\prime}$ in this category, a morphism from $f$ to $g$ is a pair $\left(F_{M}, F_{N}\right)$ of morphisms $F_{M}: M \rightarrow M^{\prime}$ and $F_{N}: N \rightarrow N^{\prime}$ satisfying $F_{N} f=f^{\prime} F_{M}$. The composition of morphisms $\left(F_{M}, F_{N}\right): f \rightarrow f^{\prime}$ and $\left(G_{M}, G_{N}\right): f^{\prime} \rightarrow f^{\prime \prime}$ is defined as $\left(G_{M} F_{M}, G_{N} F_{N}\right): f \rightarrow f^{\prime \prime}$. 
We remark that every morphism $\varphi: V \rightarrow W$ between representations of $\operatorname{rep}\left(A_{n}\right)$ is isomorphic to a morphism between direct sums of interval representations as

$$
\Phi:=\eta_{W} \varphi \eta_{V}^{-1}: \bigoplus_{1 \leq b \leq d \leq n} \mathbb{I}[b, d]^{m_{b, d}} \rightarrow \bigoplus_{1 \leq b \leq d \leq n} \mathbb{I}[b, d]^{m_{b, d}^{\prime}}
$$

in $\operatorname{arr}\left(\operatorname{rep}\left(A_{n}\right)\right)$, where

$$
\eta_{V}: V \cong \bigoplus_{1 \leq b \leq d \leq n} \mathbb{I}[b, d]^{m_{b, d}} \text { and } \eta_{W}: W \cong \bigoplus_{1 \leq b \leq d \leq n} \mathbb{I}[b, d]^{m_{b, d}^{\prime}}
$$

are indecomposable decompositions. By the following lemma, the morphism $\Phi$ can be expressed in a matrix form.

Definition 2.2 (Asashiba et al. 2019, Definition 4) The relation $\unrhd$ is defined on the set of interval representations of $A_{n}\left(\tau_{n}\right),\{\mathbb{I}[b, d] \mid 1 \leq b \leq d \leq n\}$, by setting $\mathbb{I}[a, b] \unrhd$ $\mathbb{I}[c, d]$ if and only if $\operatorname{Hom}(\mathbb{I}[a, b], \mathbb{I}[c, d])$ is nonzero. We write $\mathbb{I}[a, b] \triangleright \mathbb{I}[c, d]$ if $\mathbb{I}[a, b] \unrhd \mathbb{I}[c, d]$ and $\mathbb{I}[a, b] \neq \mathbb{I}[c, d]$.

Lemma 2.3 (Asashiba et al. 2019, Lemma 1) Let $\mathbb{I}[a, b]$ and $\mathbb{I}[c, d]$ be interval representations of $A_{n}\left(\tau_{n}\right)$.

1. The dimension of $\operatorname{Hom}(\mathbb{I}[a, b], \mathbb{I}[c, d])$ as a $K$-vector space is either 0 or 1 .

2. A $K$-vector space basis $\left\{f_{a: b}^{c: d}\right\}$ can be chosen for each $\operatorname{Hom}(\mathbb{I}[a, b], \mathbb{I}[c, d])$ such that if $\mathbb{I}[a, b] \unrhd \mathbb{I}[c, d], \mathbb{I}[c, d] \unrhd \mathbb{I}[e, f]$ and $\mathbb{I}[a, b] \unrhd \mathbb{I}[e, f]$, then

$$
f_{a: b}^{e: f}=f_{c: d}^{e: f} f_{a: b}^{c: d}
$$

The notation $[a, b]:=\{a, a+1, \ldots, b\}$ is used to denote the interval of integers $i$ with $a \leq i \leq b$. A candidate for the basis is

$$
\left(f_{a: b}^{c: d}\right)_{i}= \begin{cases}\operatorname{id}_{K} & (i \in[a, b] \cap[c, d]) \\ 0 & (\text { otherwise })\end{cases}
$$

for the case $\mathbb{I}[a, b] \unrhd \mathbb{I}[c, d]$. For convenience, we define $f_{a: b}^{c: d}=0$ for the case $\mathbb{I}[a, b] \unrhd \mathbb{I}[c, d]$. We fix this basis throughout this paper.

The morphism $\Phi$ can be written in block matrix form as

$$
\Phi=\left[\Phi_{a: b}^{c: d}\right]
$$

where each block matrix entry $\Phi_{a: b}^{c: d}: \mathbb{I}[a, b]^{m_{a, b}} \rightarrow \mathbb{I}[c, d]^{m_{c, d}^{\prime}}$ is the composition of the canonical inclusion $\iota$ with the canonical projection $\pi$ as

$$
\mathbb{I}[a, b]^{m_{a, b}} \stackrel{\iota}{\longrightarrow} \bigoplus_{1 \leq a \leq b \leq n} \mathbb{I}[a, b]^{m_{a, b}} \stackrel{\Phi}{\longrightarrow} \underset{1 \leq c \leq d \leq n}{\bigoplus_{1}} \mathbb{I}[c, d]^{m_{c, d}^{\prime}} \stackrel{\pi}{\longrightarrow} \mathbb{I}[c, d]^{m_{c, d}^{\prime}}
$$


Similarly, each block $\Phi_{a: b}^{c: d}$ can be written in matrix form with the entries in $\operatorname{Hom}(\mathbb{I}[a, b], \mathbb{I}[c, d])$ as

$$
\Phi_{a: b}^{c: d}=\left[\phi_{j}^{i}\right] \quad\left(\phi_{j}^{i} \in \operatorname{Hom}(\mathbb{I}[a, b], \mathbb{I}[c, d]), 1 \leq j \leq m_{a, b}, 1 \leq i \leq m_{c, d}^{\prime}\right) .
$$

For each relation $\mathbb{I}[a, b] \unrhd \mathbb{I}[c, d]$, according to Lemma 2.3 and factoring out $f_{a: b}^{c: d}$ from each $\phi_{j}^{i}$, one can obtain $\phi_{j}^{i}=\mu_{j}^{i} f_{a: b}^{c: d}$ for some $\mu_{j}^{i} \in K$. Similarly, factoring out $f_{a: b}^{c: d}$ from each $\Phi_{a: b}^{c: d}$, one obtains

$$
\Phi_{a: b}^{c: d}=M_{a: b}^{c: d} f_{a: b}^{c: d}
$$

where each $M_{a: b}^{c: d}$ is a $m_{c, d} \times m_{a, b}$ matrix with the entries in $K$.

Definition 2.4 Letting $\varphi$ be a morphism in $\operatorname{rep}\left(A_{n}\right)$, the block matrix form $\Phi(\varphi)$ of $\varphi$ is

$$
\Phi(\varphi)=\left[\Phi_{a: b}^{c: d}\right]=\left[M_{a: b}^{c: d} f_{a: b}^{c: d}\right] .
$$

Asashiba et al. (2019) shows that isomorphisms in the arrow category $\operatorname{arr}\left(\operatorname{rep}\left(A_{n}\right)\right)$ correspond to row and column operations in block matrix form. These operations are performed by matrix multiplication with the same restriction, i.e., the block of $\mathbb{I}[a, b] \not \mathbb{I}[c, d]$ must always be zero. The column and row operations are almost identical to those of $K$-matrices. However, because of the restriction, addition from a block to certain blocks is not permissible.

We can discuss column operations next. We define a morphism $\Phi^{\prime}$ such that the following diagram commutes:

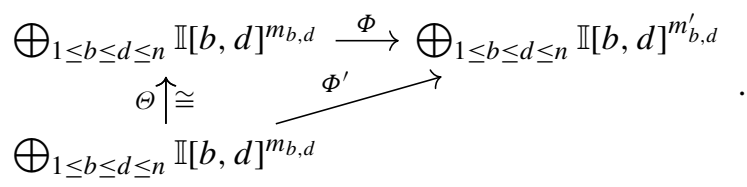

Therein, $\Theta$ is an isomorphism: $\Phi$ and $\Phi^{\prime}$ are isomorphic in the arrow category. Observing that the domain and codomain of $\Theta$ are direct summations, then $\Theta$ can also be written in a block matrix form $\left[C_{a: b}^{c: d} f_{a: b}^{c: d}\right]$ by an argument similar to that made for $\Phi$. The multiplication $\Phi \Theta$ denotes column operations on $\Phi$. Its block at column $a: b$ and row $c: d$ is

$$
\begin{aligned}
{[\Phi \Theta]_{a: b}^{c: d} } & =\sum_{\mathbb{I}[a, b] \unrhd \mathbb{I}[e, f] \unrhd \mathbb{I}[c, d]}\left(M_{e: f}^{c: d} f_{e: f}^{c: d}\right)\left(C_{a: b}^{e: f} f_{a: b}^{e: f}\right) \\
& =\left(\sum_{\mathbb{I}[a, b] \unrhd \mathbb{I}[e, f] \unrhd \mathbb{I}[c, d]} M_{e: f}^{c: d} C_{a: b}^{e: f}\right) f_{a: b}^{c: d} .
\end{aligned}
$$

The difference from the usual column operations on $K$-matrices is the part not only

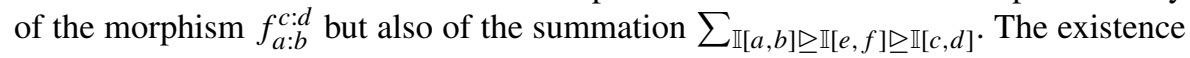


of the morphism $f_{a: b}^{c: d}$ merely means that the block with $\mathbb{I}[a, b] \unrhd \mathbb{I}[c, d]$ must always

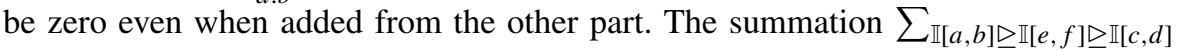
might be somewhat more complicated. It means that not all the column operations are permissible: only the following cases.

- Elementary column operations (switching, multiplication, and addition) within the same interval are always permissible.

- Column addition to $\mathbb{I}[a, b]$ from another interval $\mathbb{I}[e, f]$ with relation $\mathbb{I}[a, b] \triangleright$ $\mathbb{I}[e, f]$ is permissible.

Similar properties of permissibility hold for row operations.

- Elementary row operations (switching, multiplication, and addition) within the same interval are always permissible.

- Row addition to $\mathbb{I}[a, b]$ from another interval $\mathbb{I}[e, f]$ with relation $\mathbb{I}[e, f] \triangleright \mathbb{I}[a, b]$ is permissible.

The following is an example of permissibility in the case of $\operatorname{arr}\left(\operatorname{rep}\left(A_{3}(b f)\right)\right)$.

Example 2.5 The following matrix is the block matrix form of $\operatorname{arr}\left(\operatorname{rep}\left(A_{3}(b f)\right)\right)$, for which we use the symbols $a: b$ to denote the rows and columns corresponding to the direct summands $\mathbb{I}[a, b]^{m_{a, b}}$. Each block $M_{a: b}^{c: d} f_{a: b}^{c: d}$ is abbreviated to $*$ if $f_{a: b}^{c: d} \neq$ 0 and $\emptyset$ otherwise. The prohibited additions for columns and rows, written as red arrows, correspond to the positions of the zero blocks $\emptyset$. Because the column addition from lower to upper blocks and row addition from left to right blocks are always prohibited, we write down only the prohibited column additions from upper to lower, and prohibited row addition from right to left. A notable fact is that either $f_{a: b}^{1: 3}$ or $f_{1: 3}^{a: b}$ is 0 for arbitrary $(a, b) \neq(1,3)$. In other words, $\mathbb{I}[1,3] \unrhd \mathbb{I}[a, b] \unrhd \mathbb{I}[1,3]$ if and only if $(a, b)=(1,3)$. We refer to this fact in the proof of Lemma 4.4.

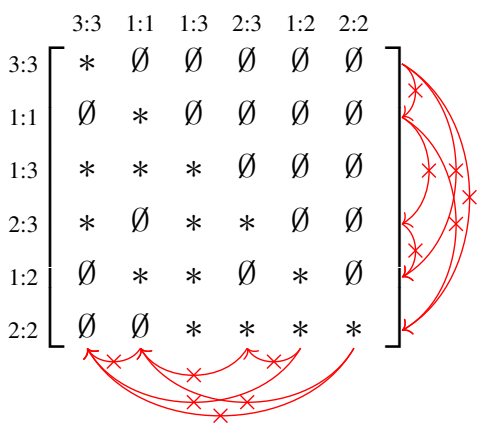

\section{Induced maps via quiver representations}

For discussion presented in this section, we redefine the induced map of a correspondence using quiver representations. Let $H=H(-; K)$ be the homology functor with coefficients in $K$. As a representation of an $A_{3}(b f)$ type quiver, the diagram 
Fig. 3 Overview of our definition of the induced map $F_{*}$ of a correspondence. The isomorphism from the first row to the second row is an indecomposable decomposition. The inclusion map on the right-hand side is the canonical injection of the vector space

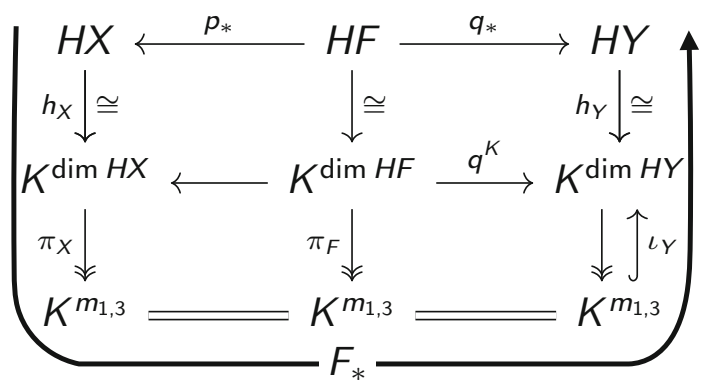

$H X \stackrel{p_{*}}{\leftarrow} H F \stackrel{q_{*}}{\rightarrow} H Y$ induced by a correspondence $F \subset X \times Y$ can be decomposed into a direct sum of interval representations as

$$
h:\left(H X \stackrel{p_{*}}{\leftarrow} H F \stackrel{q_{*}}{\rightarrow} H Y\right) \stackrel{\cong}{\rightarrow} \bigoplus_{1 \leq b \leq d \leq 3} \mathbb{I}[b, d]^{m_{b, d}} .
$$

This indecomposable decomposition can be written as the following diagram:

$$
\begin{aligned}
& H X \longleftarrow p_{*} H \stackrel{q_{*}}{\longrightarrow} H Y \\
& \bigoplus_{1 \leq b \leq d \leq 3} \mathbb{I}[b, d]^{m_{b, d}}=\quad K^{\operatorname{dim} H X} \longleftarrow K^{\operatorname{dim} H F} \stackrel{q^{K}}{\longrightarrow} K^{\operatorname{dim} H Y} .
\end{aligned}
$$

The choice of bases gives us a relation between bases of $H X$ and $H Y$, which can be regarded as a map from $H X$ to $H Y$. For example, an interval representation $\mathbb{I}[1,2]$ assigns an element of the standard basis of $K^{\operatorname{dim} H X}$ to 0 in $K^{\operatorname{dim} H Y}$. Therefore, nontrivial assignment occurs only with the interval representations $\mathbb{I}[1,3]=\left(K \stackrel{\mathrm{id}_{K}}{\leftarrow} K \stackrel{\mathrm{id}_{K}}{\rightarrow}\right.$ $K)$. By regarding the other interval representations as 0 maps from $H X$ to $H Y$, one can define a map $\iota_{Y} \circ \pi_{X}: K^{\operatorname{dim} H X} \rightarrow K^{\operatorname{dim} H Y}$ factoring the interval $\mathbb{I}[1,3]$ as

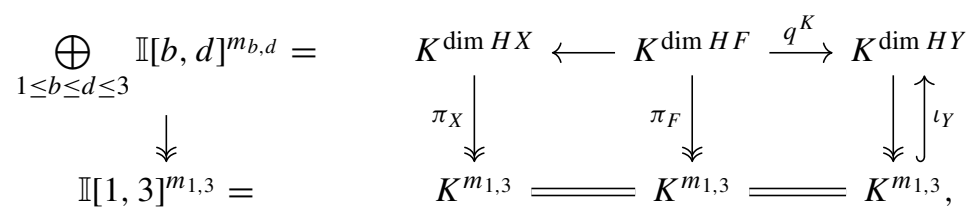

where the arrows $\rightarrow$ are the canonical projections of the vector spaces. The morphism $\iota_{Y}$ is the canonical injection of the vector space. Composing the path of morphisms, one can define the induced map of $F$ through $h$ as

$$
F_{*}:=h_{Y}^{-1} \circ \iota_{Y} \circ \pi_{X} \circ h_{X}: H X \rightarrow H Y .
$$

This definition requires no two assumptions described in Definition 1.3. Although our definition depends on the choice of isomorphism of indecomposable decomposi- 
tion, when the two assumptions are satisfied, our definition coincides with the original definition $q_{*} \circ p_{*}^{-1}$ by the following theorem.

Theorem 3.1 Define $F_{*}:=h_{Y}^{-1} \circ \iota_{Y} \circ \pi_{X} \circ h_{X}$. If $F$ is homologically complete and homologically consistent, then $F_{*}=q_{*} \circ p_{*}^{-1}$.

Proof The claim to prove is

$$
q_{*} \circ p_{*}^{-1}=h_{Y}^{-1} \circ \iota_{Y} \circ \pi_{X} \circ h_{X} .
$$

Since the morphism $p_{*}$ is surjective, this is equivalent to

$$
q_{*} \circ p_{*}^{-1} \circ p_{*}=h_{Y}^{-1} \circ \iota_{Y} \circ \pi_{X} \circ h_{X} \circ p_{*} .
$$

In addition, $q_{*}=q_{*} \circ p_{*}^{-1} \circ p_{*}$ because of the homological consistency $q_{*}\left(\operatorname{Ker}\left(p_{*}\right)\right)=$ 0 . Therefore, what must be proven is

$$
q_{*}=h_{Y}^{-1} \circ \iota_{Y} \circ \pi_{X} \circ h_{X} \circ p_{*} .
$$

By chasing the diagram of Fig. 3, this equation results in

$$
q^{K}=\iota_{Y} \circ \pi_{F}
$$

The standard basis of $K^{\operatorname{dim} H F}$ corresponds to the standard bases of the four intervals $\mathbb{I}[2,3], \mathbb{I}[2,2], \mathbb{I}[1,2]$, and $\mathbb{I}[1,3]$. Here we remark that the homological consistency $q_{*}\left(\operatorname{Ker}\left(p_{*}\right)\right)=0$ is equivalent to $m_{2,3}=0$. That is to say that $\mathbb{I}[2,3]$ does not exist as a direct summand. Moreover, the basis corresponding to $\mathbb{I}[2,2]$ and $\mathbb{I}[1,2]$ is mapped to 0 by both $q^{K}$ and $\iota_{Y} \circ \pi_{F}$. The definition clarifies that $q^{K}(a)=\iota_{Y} \circ \pi_{F}(a)$ holds for each element $a$ of the standard basis of $K^{\operatorname{dim} H F}$ corresponding to the standard bases of $\mathbb{I}[1,3]$.

\section{Persistence analysis for sampled maps}

The ability to decompose and emphasize specifically the interval representation $\mathbb{I}[1,3]$ provides persistence analysis for sampled maps. One can consider the following problem, which is similar to Problem 1.1 but which requires additional assumptions of embeddings.

Problem 4.1 Let $f: X \rightarrow Y$ be a continuous map for $X, Y \subset \mathbb{R}^{n}$. If $X, Y$, and $f$ are unknown, then we know only a sampled map $f \uparrow_{S}: S \rightarrow f(S)$, which is a restriction of $f$ on a finite subset $S \subset X$, then can we retrieve any information about the homology-induced map $f_{*}: H X \rightarrow H Y$ ?

It is noteworthy that sampling $S$ is a point cloud capturing some topological features of $X$ when $S$ is sufficiently dense. Originally, after Edelsbrunner et al. (2015) set this problem with the additional assumption that $X=Y$, they constructed a persistent 
homology of eigenspaces of the sampled map to analyze the eigenspaces of the discrete dynamical system $f$.

In this section, we explain how to construct another persistent homology of a sampled map, which captures the generator of $H X$ and $H f(X)$ connected by $f$. We can use filtrations of two types in Sects. 4.1 and 4.2. The former filtration is generated using simplicial complexes. Then we can prove a stability theorem (Theorem 5.6) of this construction in Sect. 5. The latter filtration, which is generated using grids as in Sect. 3, also derives stability (Theorem 5.8). The stability theorem for the latter, however, requires more assumptions than for the former. For that reason, we explain it in this order.

\subsection{Construction using simplicial complexes}

First, we generate a filtration of abstract simplicial complexes of $S$ as

$$
C_{1} \subset C_{2} \subset \cdots \subset C_{\ell}
$$

each simplex of which has elements of $S$ as its vertices, so that the filtration can capture the topology of the underlying space $X$. For example, Čech complexes or Vietoris-Rips complexes (Edelsbrunner and Harer 2010) are available.

Definition 4.2 Let $P$ be a point cloud in $\mathbb{R}^{n}$. The $\check{C}$ ech complex $\Gamma_{r}$ for $P$ with a radius $r$ is the abstract simplicial complex defined as

$$
\Gamma_{r}=\left\{\sigma \subset P \mid \bigcap_{p \in \sigma} B(p ; r) \neq \emptyset\right\}
$$

where $B(p ; r)$ is the closed ball of center $p$ and radius $r$. Letting $d_{\mathbb{R}^{n}}$ be the Euclidean metric on $\mathbb{R}^{n}$, then the Vietoris-Rips complex $V_{r}$ for $P$ with a radius $r$ is the abstract simplicial complex defined as

$$
V_{r}=\left\{\sigma \subset P \mid \forall p_{1}, p_{2} \in \sigma, d_{\mathbb{R}^{n}}\left(p_{1}, p_{2}\right) \leq 2 r\right\}
$$

Similarly, we generate a filtration of abstract simplicial complexes

$$
D_{1} \subset D_{2} \subset \cdots \subset D_{\ell}
$$

for $f(S)$.

Using these filtrations, we attempt to build a filtration of maps from $f \uparrow_{S}$ to analyze the persistence of the original map $f$, in analogy with the classical technique of persistent homology. Although we expect the sampled map to derive a simplicial map $C_{i} \rightarrow D_{i}$ on each $i$-th filter, in general, they can derive only a simplicial partial map $f_{i}: C_{i} \nrightarrow D_{i}$. For a simplex $\left\{s_{1}, \ldots, s_{d}\right\} \in C_{i}$, it is not assumed that

\footnotetext{
${ }^{1}$ A correspondence $F$ from $X$ to $Y$ is a partial map if $F(x)$ is a singleton or empty set for all $x \in X$, where $F(x):=\{y \in Y \mid(x, y) \in F\}$.
} 
$\left\{f\left(s_{1}\right), \ldots, f\left(s_{d}\right)\right\} \in D_{i}$. Therefore, the conventional technique computing topological persistence for simplicial maps (Dey et al. 2014) is not available in this setup.

We remark that the graph $\operatorname{Gr}\left(f\left\lceil_{S}\right)=\left\{(s, f(s)) \in \mathbb{R}^{n} \times \mathbb{R}^{n} \mid s \in S\right\}\right.$ of the sampled map is a point cloud in $\mathbb{R}^{n} \times \mathbb{R}^{n}$. Let us define the $i$-th abstract simplicial complex $G_{i}$ of $\operatorname{Gr}\left(f \uparrow_{S}\right)$ as

$$
\begin{aligned}
G_{i} & :=\left\{\{ ( s _ { 1 } , f ( s _ { 1 } ) ) , \ldots , ( s _ { d } , f ( s _ { d } ) ) \} \subset \operatorname { G r } \left(f\left\lceil_{S}\right) \mid\left\{s_{1}, \ldots, s_{d}\right\} \in C_{i},\right.\right. \\
& \left.\left\{f\left(s_{1}\right), \ldots, f\left(s_{d}\right)\right\} \in D_{i}\right\} .
\end{aligned}
$$

One can show that $\left\{G_{i}\right\}$ forms a filtration. The sequence of the partial maps can be regarded as a sequence $\left\{C_{i} \stackrel{p_{i}}{\leftarrow} G_{i} \stackrel{q_{i}}{\rightarrow} D_{i}\right\}$ of pairs of canonical projections $\left(p_{i}, q_{i}\right)$. This sequence of pairs constitutes a filtration induced by the inclusion maps of the filtration

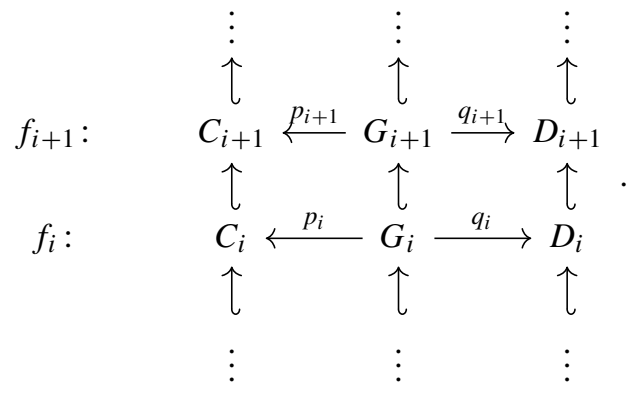

By applying the homology functor to the sequence, one can obtain a sequence of representations of the $A_{3}(b f)$ type quiver as

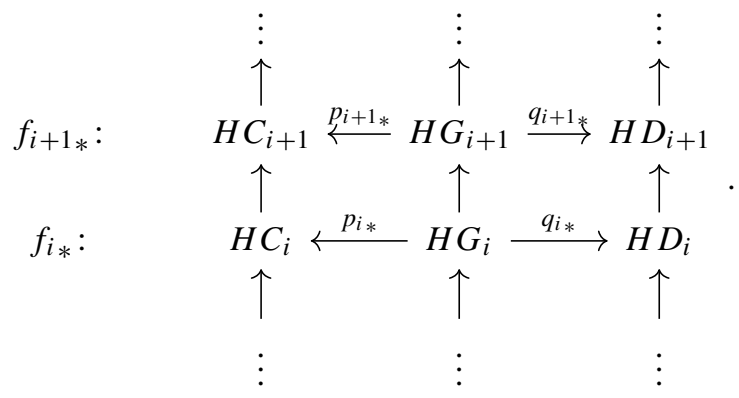

Remark 4.3 Earlier research (Edelsbrunner et al. 2015) used domains of partial maps to construct a similar filtration. For a partial map $f_{i}: C_{i} \nrightarrow D_{i}{ }^{2}$, the domain $\operatorname{dom} f_{i}$ is defined as

$$
\operatorname{dom} f_{i}:=\left\{\left\{s_{1}, \ldots, s_{d}\right\} \in C_{i} \mid\left\{f\left(s_{1}\right), \ldots, f\left(s_{d}\right)\right\} \in D_{i}\right\} .
$$

\footnotetext{
2 To be accurate, the earlier research considers only the case in which $C_{i}=D_{i}$ to analyze self-maps. 
Using the inclusion $\iota_{i}$ : $\operatorname{dom} f_{i} \hookrightarrow C_{i}$ and the simplicial map $f_{i}^{\prime}: \operatorname{dom} f_{i} \rightarrow D_{i}$ induced by the map $f$ on $\operatorname{dom} f_{i}$, the domains induce the representation

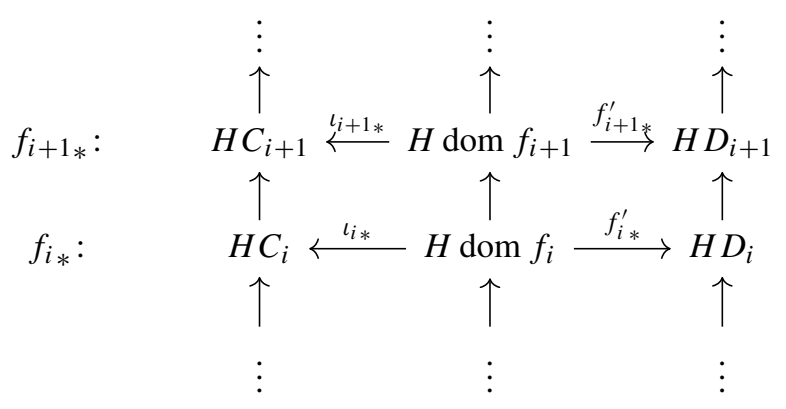

similarly. By the definitions of $G_{i}$ and $\operatorname{dom} f_{i}$, it is readily apparent that the induced representation (3) is isomorphic to our representation (2). For consistency from the viewpoint of graphs and correspondences, we adopt the simplicial complexes $\left\{G_{i}\right\}$.

Here, decomposing each filter $H C_{i} \leftarrow H G_{i} \rightarrow H D_{i}$ as a representation to the intervals $\bigoplus_{1 \leq b \leq d \leq 3} \mathbb{I}[b, d]^{m_{b, d}^{i}}$, the representation (2) is isomorphic to

$$
\Lambda: \bigoplus_{1 \leq b \leq d \leq 3} \mathbb{I}[b, d]^{m_{b, d}^{1}} \rightarrow \bigoplus_{1 \leq b \leq d \leq 3} \mathbb{I}[b, d]^{m_{b, d}^{2}} \rightarrow \cdots \rightarrow \bigoplus_{1 \leq b \leq d \leq 3} \mathbb{I}[b, d]^{m_{b, d}^{\ell}}
$$

Projecting to $\mathbb{I}[1,3]$ again yields a sequence of subrepresentations as

$$
\Lambda[1,3]: \mathbb{I}[1,3]^{m_{1,3}^{1}} \rightarrow \mathbb{I}[1,3]^{m_{1,3}^{2}} \rightarrow \cdots \rightarrow \mathbb{I}[1,3]^{m_{1,3}^{\ell}}
$$

One must carefully construct $\Lambda[1,3]$. We write canonical projections and injections defined by direct sum respectively as

$$
\begin{aligned}
& \pi_{i}: \bigoplus_{1 \leq b \leq d \leq 3} \mathbb{I}[b, d]^{m_{b, d}^{i}} \rightarrow \mathbb{I}[1,3]^{m_{1,3}^{i}} \\
& \iota_{i}: \mathbb{I}[1,3]^{m_{1,3}^{i}} \rightarrow \bigoplus_{1 \leq b \leq d \leq 3} \mathbb{I}[b, d]^{m_{b, d}^{i}},
\end{aligned}
$$

and the morphisms in $\Lambda$ as

$$
\Phi_{i}: \bigoplus_{1 \leq b \leq d \leq 3} \mathbb{I}[b, d]^{m_{b, d}^{i}} \rightarrow \bigoplus_{1 \leq b \leq d \leq 3} \mathbb{I}[b, d]^{m_{b, d}^{i+1}}
$$

Then, the morphisms in $\Lambda[1,3]$

$$
\Phi_{i} 1: 3: \mathbb{I}[1,3]^{m_{1,3}^{i}} \rightarrow \mathbb{I}[1,3]^{m_{1,3}^{i+1}}
$$


are defined as

$$
\Phi_{i 1: 3}^{1: 3}:=\pi_{i+1} \circ \Phi_{i} \circ \iota_{i},
$$

which is the submatrix at $(1: 3,1: 3)$ in a block matrix form of $\Phi_{i}$.

At a glance, this construction seems natural, but " $\pi: \Lambda \rightarrow \Lambda[1,3]$ " is not a morphism in the representation category. In other words,

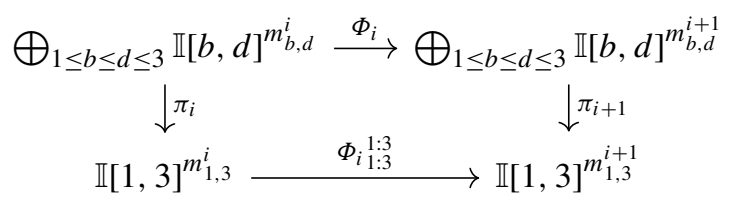

does not always commute. Consequently, the choice of isomorphism of indecomposable decomposition on each filter $H C_{i} \leftarrow H G_{i} \rightarrow H D_{i}$ might make a difference in the output persistence diagram. To make sense of this analysis, the output persistence diagram should be determined uniquely and independently of the choice of isomorphism. Theorem 4.6 guarantees uniqueness and independence. Before the theorem can be stated, two lemmas must be introduced.

The restriction to the block $(1: 3,1: 3)$ has the following functoriality.

Lemma 4.4 Let

$$
\Theta: \bigoplus_{1 \leq b \leq d \leq 3} \mathbb{I}[b, d]^{m_{b, d}^{1}} \rightarrow \bigoplus_{1 \leq b \leq d \leq 3} \mathbb{I}[b, d]^{m_{b, d}^{2}}
$$

and

be block matrix forms, then

$$
\Psi: \bigoplus_{1 \leq b \leq d \leq 3} \mathbb{I}[b, d]^{m_{b, d}^{2}} \rightarrow \bigoplus_{1 \leq b \leq d \leq 3} \mathbb{I}[b, d]^{m_{b, d}^{3}}
$$

$$
[\Psi \Theta]_{1: 3}^{1: 3}=\Psi_{1: 3}^{1: 3} \Theta_{1: 3}^{1: 3} .
$$

Proof Let the corresponding scalar matrix symbols of $\Theta$ and $\Psi$ respectively be $M$ and $N$. The block matrix of $\Psi \Theta$ at $(1: 3,1: 3)$ is

$$
\begin{aligned}
{[\Psi \Theta]_{1: 3}^{1: 3} } & =\sum_{\mathbb{I}[1,3] \unrhd \mathbb{I}[a, b] \unrhd \mathbb{I}[1,3]}\left(N_{a: b}^{1: 3} f_{a: b}^{1: 3}\right)\left(M_{1: 3}^{a: b} f_{1: 3}^{a: b}\right) \\
& =\left(\sum_{\mathbb{I}[1,3] \unrhd \mathbb{I}[a, b] \unrhd \mathbb{I}[1,3]} N_{a: b}^{1: 3} M_{1: 3}^{a: b}\right) f_{1: 3}^{1: 3},
\end{aligned}
$$

but only $\mathbb{I}[1,3]$ can be a candidate for the interval $\mathbb{I}[a, b]$ as described in Example 2.5. Therefore,

$$
\begin{aligned}
{[\Psi \Theta]_{1: 3}^{1: 3} } & =N_{1: 3}^{1: 3} M_{1: 3}^{1: 3} f_{1: 3}^{1: 3} \\
& =\left(N_{1: 3}^{1: 3} f_{1: 3}^{1: 3}\right)\left(M_{1: 3}^{1: 3} f_{1: 3}^{1: 3}\right) \\
& =\Psi_{1: 3}^{1: 3} \Theta_{1: 3}^{1: 3} .
\end{aligned}
$$


Lemma 4.5 Let $\Theta: \bigoplus_{1 \leq b \leq d \leq 3} \mathbb{I}[b, d]^{m_{b, d}^{1}} \rightarrow \bigoplus_{1 \leq b \leq d \leq 3} \mathbb{I}[b, d]^{m_{b, d}^{2}}$ be the block matrix form of an isomorphism. Then $\Theta_{1: 3}^{1: 3}$ is an isomorphism.

Proof Let $\Psi$ be the inverse of $\Theta$. By Lemma 4.4,

$$
[\Psi \Theta]_{1: 3}^{1: 3}=\Psi_{1: 3}^{1: 3} \Theta_{1: 3}^{1: 3}
$$

The left-hand side is the block $(1: 3,1: 3)$ of the identity map, which is the identity map on $\mathbb{I}[1,3]^{m_{1,3}^{1}}$. Similarly, $\Theta_{1: 3}^{1: 3} \Psi_{1: 3}^{1: 3}$ is the identity map on $\mathbb{I}[1,3]^{m_{1,3}^{2}}$. Therefore, it follows that $\Theta_{1: 3}^{1: 3}$ is isomorphic.

Theorem 4.6 The isomorphism class of

$$
\Phi_{i} i_{1: 3}^{1: 3}: \mathbb{I}[1,3]^{m_{1,3}^{i}} \rightarrow \mathbb{I}[1,3]^{m_{1,3}^{i+1}}
$$

is determined uniquely and independently of the choice of the bases of

$$
\Phi_{i}: \bigoplus_{1 \leq b \leq d \leq 3} \mathbb{I}[b, d]^{m_{b, d}^{i}} \rightarrow \bigoplus_{1 \leq b \leq d \leq 3} \mathbb{I}[b, d]^{m_{b, d}^{i+1}}
$$

Proof Let $\Psi_{i}$ be a morphism isomorphic to $\Phi_{i}$, which is written as a commutative diagram of

$$
\begin{array}{cc}
\bigoplus_{1 \leq b \leq d \leq n} \mathbb{I}[b, d]^{m_{b, d}} \stackrel{\Phi_{i}}{\longrightarrow} \bigoplus_{1 \leq b \leq d \leq n} \mathbb{I}[b, d]^{m_{b, d}^{\prime}} \\
C \downarrow \cong \\
R \uparrow \cong \\
\bigoplus_{1 \leq b \leq d \leq n} \mathbb{I}[b, d]^{m_{b, d}} \stackrel{\Psi_{i}}{\longrightarrow} \bigoplus_{1 \leq b \leq d \leq n} \mathbb{I}[b, d]^{m_{b, d}^{\prime}}
\end{array}
$$

for some isomorphisms $C$ and $R$. That is, $\Phi_{i}=R \Psi_{i} C$; by Lemma 4.4, applying the restriction yields

$$
\begin{aligned}
\Phi_{i 1: 3}^{1: 3} & =\left[R \Psi_{i} C\right]_{1: 3}^{1: 3} \\
& =R_{1: 3}^{1: 3} \Psi_{i}^{1: 3} C_{1: 3}^{1: 3} .
\end{aligned}
$$

By Lemma $4.5, R_{1: 3}^{1: 3}$ and $C_{1: 3}^{1: 3}$ are isomorphisms. Therefore, $\Phi_{i 1: 3}^{1: 3}$ is isomorphic to $\Psi_{i}^{1: 3}$.

Because consideration of $\mathbb{I}[1,3]=\left(K \stackrel{\mathrm{id}_{K}}{\leftarrow} K \stackrel{\mathrm{id}_{K}}{\rightarrow} K\right)$ as $K$ omits no information, the sequence $\Lambda[1,3]$ can be regarded as a sequence of vector spaces

$$
K^{m_{1,3}^{1}} \rightarrow K^{m_{1,3}^{2}} \rightarrow \cdots \rightarrow K^{m_{1,3}^{\ell}}
$$

which is an $A_{\ell}$ type representation. 
Definition 4.7 For a sampled map $f \uparrow_{S}$, persistent homology of the sampled map $f \uparrow_{S}$ is the representation (4).

Decomposing the persistent homology into intervals, one can draw a persistence diagram that shows us the length of the generators of homology in both filtrations, which are assigned by $f$. Simultaneously, we have constructed the filtration of complexes approximating unknown spaces $X$ and $f(X)$.

Comparison with earlier research This persistence diagram provides no information about eigenvectors, unlike that provided by Edelsbrunner et al. (2015). Nevertheless, it is widely applicable. First, because our method uses no eigenspace functor, we need not require both sides' spaces to be the same. Therefore, even in the case of sampled dynamical systems $X=Y$, similarly to earlier research, we can weaken the assumption $f\left\lceil_{S}: S \rightarrow S\right.$ to $f \uparrow_{S}: S \rightarrow f(S)$ and take another filtration on $f(S)$. (If $f(S)$ is insufficiently dense for sampling $X$, then we can take $S \cup f(S)$ instead.) Moreover, because the previous method must set an eigenvalue before analysis, they must predict some behavior of $f$ in advance. By contrast, our method requires no prior information. The numerical experiments in Sect. 7 emphasize this difference.

\subsection{Construction using a grid}

The construction of the persistent homology discussed in Sect. 4.1 is applicable to the filtrations generated by expanding a graph. This perspective provides a persistent approach to correspondences of sampled maps constructed by dividing the spaces (Harker et al. 2016). Presuming that spaces $X$ and $Y$ are embedded into Euclidean space $\mathbb{R}^{n}$, and that both $\mathbb{R}^{n}$ are divided by $n$-dimensional $\varepsilon$-cubes, then

$$
\left\{\left[a_{1} \varepsilon,\left(a_{1}+1\right) \varepsilon\right] \times \cdots \times\left[a_{n} \varepsilon,\left(a_{n}+1\right) \varepsilon\right] \mid a_{1}, \ldots, a_{n} \in \mathbb{Z}\right\} .
$$

To distinguish the two divisions, we express this set as $\mathcal{X}_{\varepsilon}$ for the $X$-side Euclidean space and $\mathcal{Y}_{\varepsilon}$ for $Y$-side. Letting $f \uparrow_{S}$ be a sampled map of a continuous map $f: X \rightarrow$ $Y$, and letting $p$ and $q$ be the respective canonical projections of $\mathbb{R}^{n} \times \mathbb{R}^{n}$ to the $X$-side and $Y$-side Euclidean spaces, then we generate a correspondence

$$
\begin{aligned}
F_{\varepsilon}^{f \uparrow_{S}}:=\left\{(x, y) \in \mathbb{R}^{n} \times \mathbb{R}^{n} \mid x\right. & \in \exists X^{\prime} \in \mathcal{X}_{\varepsilon}, \\
y & \in \exists Y^{\prime} \in \mathcal{Y}_{\varepsilon},\left(X^{\prime} \times Y^{\prime}\right) \cap \operatorname{Gr}\left(f\left\lceil_{S}\right) \neq \emptyset\right\},
\end{aligned}
$$

where $\operatorname{Gr}\left(f \uparrow_{S}\right):=\{(s, f(s)) \mid s \in S\}$ (see Fig. 2).

We use the $L^{\infty}$ metric defined as explained below.

Definition 4.8 The $L^{\infty}$ metric $d_{\infty}$ on $\mathbb{R}^{n}\left(\right.$ or $\left.\mathbb{R}^{n} \times \mathbb{R}^{n}\right)$ is defined as

$$
d_{\infty}(x, y):=\max _{i}\left(\left|x_{i}-y_{i}\right|\right)
$$

for all $x=\left(x_{i}\right), y=\left(y_{i}\right) \in \mathbb{R}^{n}$ (or $\mathbb{R}^{n} \times \mathbb{R}^{n}$, respectively). 
It is noteworthy that we abuse the same symbol $d_{\infty}$ for both spaces $\mathbb{R}^{n}$ and $\mathbb{R}^{n} \times$ $\mathbb{R}^{n}$. To construct a filtration along with the grids, one can define the filtration of a correspondence as

$$
F_{i \varepsilon}:=\left(F_{\varepsilon}^{f\lceil s}\right)_{i \varepsilon}=\left\{r \in \mathbb{R}^{n} \times \mathbb{R}^{n} \mid d_{\infty}\left(r, F_{\varepsilon}^{f\lceil s}\right) \leq i \varepsilon\right\} \quad\left(i \in \mathbb{Z}_{\geq 1}\right),
$$

and morphisms $p_{i \varepsilon}:=p \uparrow_{F_{i \varepsilon}}$ and $q_{i \varepsilon}:=q \uparrow_{F_{i \varepsilon}}$. Here we restrict $i=1, \ldots, \ell$ for sufficiently large $\ell$. Consequently, we have a similar diagram to that presented before, as

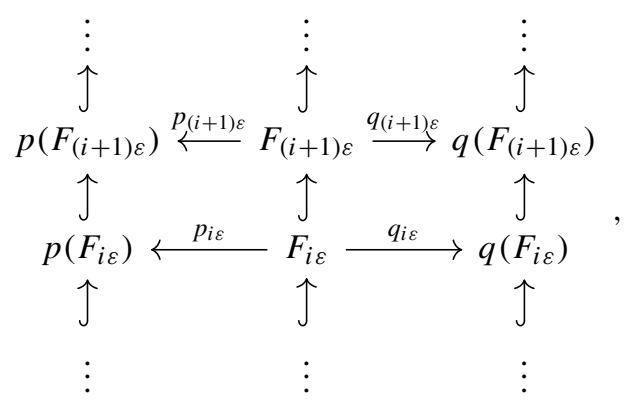

thereby allowing us to obtain the filtrations $\left\{p\left(F_{i \varepsilon}\right)\right\}$ and $\left\{q\left(F_{i \varepsilon}\right)\right\}$, capturing the persistent topological features of $X$ and $f(X)$. Again, the homology functor derives the sequence of morphisms in $\operatorname{rep}\left(A_{3}(b f)\right)$. Therefore, we can execute the same analysis as before, transforming it into block matrix form, restricting it to the blocks (1:3, 1:3), identifying it with a representation of the $A_{\ell}$ type quiver, and consequently producing a persistence diagram.

\section{Stability}

For a tool in topological data analysis to be regarded as practical, the output persistence diagrams should behave continuously as a function of input data. Such a property, known as stability (Cohen-Steiner et al. 2007; Chazal et al. 2009), has been proved for persistence modules on $\mathbb{R}$.

Let vect be the category of finite-dimensional vector spaces, $\mathbb{R}$ be the poset category of real numbers ${ }^{3}$. An object of the functor category vect ${ }^{\mathbb{R}}$ is also called a persistence module in some papers. To distinguish it from our definition, we call this an $\mathbb{R}$ persistence module.

Specifically, for an $\mathbb{R}$-persistence module $M$, we assign a vector space $M_{t}$ for $t \in \mathbb{R}$ and a linear map $\varphi_{M}(s, t): M_{s} \rightarrow M_{t}$ for $s \leq t \in \mathbb{R}$, where

$$
\varphi_{M}(t, t)=\operatorname{id}_{M_{t}} \text { and } \varphi_{M}(s, t) \circ \varphi_{M}(r, s)=\varphi_{M}(r, t)
$$

\footnotetext{
${ }^{3}$ For $x, y \in \mathbb{R}$, a morphism $x \rightarrow y$ uniquely exists if and only if $x \leq y$.
} 
for all $r \leq s \leq t \in \mathbb{R}$. We designate the linear maps $\varphi_{M}(s, t)$ as transition maps. A morphism $f: M \rightarrow N$ of $\mathbb{R}$-persistence modules is a natural transformation: a collection of morphisms $\left\{f_{t}: M_{t} \rightarrow N_{t} \mid t \in \mathbb{R}\right\}$ assuming the diagrams

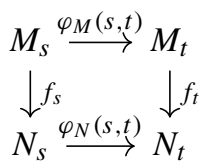

commute for all $s \leq t \in \mathbb{R}$.

It is noteworthy that every persistence module can be regarded similarly as a functor from a finite poset category to vect.

The fundamental objects of $\mathbb{R}$-persistence modules are interval modules $K_{I}$ for intervals $I \subset \mathbb{R}$, given as $\left(K_{I}\right)_{t}=K$ for $t \in I$ and $\left(K_{I}\right)_{t}=0$ otherwise, and with the morphism corresponding to $s \leq t \in I$ is an identity map. As is the case with persistent homology, every $\mathbb{R}$-persistence module can be decomposed into a direct sum of interval modules (Crawley-Boevey 2015).

A distance between $\mathbb{R}$-persistence modules can be defined as the interleaving distance.

Definition 5.1 For $\delta \geq 0$, define the functor $(\cdot)(\delta):$ vect $^{\mathbb{R}} \rightarrow$ vect $^{\mathbb{R}}$, called the shift functor, as explained below. For an $\mathbb{R}$-persistence module $M, M(\delta)_{t}:=M_{t+\delta}$ and $\varphi_{M(\delta)}(s, t):=\varphi_{M}(s+\delta, t+\delta)$. For a morphism $f$ in $\operatorname{vect}^{\mathbb{R}}, f(\delta):=f_{t+\delta}$.

Definition 5.2 For an $\mathbb{R}$-persistence module $M$ and $\delta \geq 0$, the $\delta$-transition morphism $\varphi_{M}(\delta): M \rightarrow M(\delta)$ is defined as $\varphi_{M}(\delta)_{t}:=\varphi_{M}(t, t+\delta)$.

Definition 5.3 $\mathbb{R}$-persistence modules $M$ and $N$ are said to be $\delta$-interleaved if there exist morphisms $f: M \rightarrow N(\delta)$ and $g: N \rightarrow M(\delta)$ such that

$$
g(\delta) \circ f=\varphi_{M}(2 \delta) \text { and } f(\delta) \circ g=\varphi_{N}(2 \delta) .
$$

The interleaving distance $d_{I}$ : vect ${ }^{\mathbb{R}} \times$ vect $^{\mathbb{R}} \rightarrow[0, \infty]$ is defined as

$$
d_{I}(M, N):=\inf _{\delta}\{M \text { and } N \text { are } \delta \text {-interleaved }\}
$$

An often used distance between persistence diagrams is the bottleneck distance defined by bijections between them. The interleaving distance of $\mathbb{R}$-persistence modules is well-known to be equal to the bottleneck distance of their persistence diagrams (Lesnick 2015; Bauer and Lesnick 2014). Consequently, by showing that a distance between input data is greater than the interleaving distance of their $\mathbb{R}$ persistence module, one can prove the stability of the persistence diagrams as a function of the input data.

In analogy with Edelsbrunner et al. (2015), stability theorems for some filtrations also hold on our analysis. The discrete setting discussed in Sect. 4 is sufficient for implementation, but we extend it to a continuous analysis to prove its stability. 


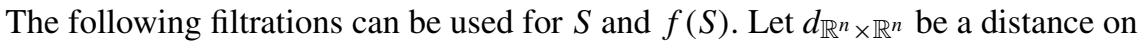
$\mathbb{R}^{n} \times \mathbb{R}^{n}$ defined as

$$
d_{\mathbb{R}^{n} \times \mathbb{R}^{n}}\left(\left(x_{1}, y_{1}\right),\left(x_{2}, y_{2}\right)\right):=\max \left\{d_{\mathbb{R}^{n}}\left(x_{1}, x_{2}\right), d_{\mathbb{R}^{n}}\left(y_{1}, y_{2}\right)\right\},
$$

where $d_{\mathbb{R}^{n}}$ is the Euclidean metric on $\mathbb{R}^{n}$. For a subset $U$ of $\mathbb{R}^{n}$, we define a function $d_{U}: \mathbb{R}^{n} \rightarrow \mathbb{R}_{\geq 0}$ to be the infimum distance to a point in $U$. Similarly, we abuse the same symbol $\bar{d}_{U}: \mathbb{R}^{n} \times \mathbb{R}^{n} \rightarrow \mathbb{R}_{\geq 0}$ for a subset $U$ of another space $\mathbb{R}^{n} \times \mathbb{R}^{n}$. We use the notation $U_{r}:=d_{U}^{-1}[0, r]$ to denote the sublevel sets.

Let Top ${ }^{(\mathrm{bf})}$ be the functor category from the $A_{3}(b f)$ type quiver $(\cdot \leftarrow \cdot \rightarrow \cdot)$ as a poset category to the category of topological spaces. The sublevel sets $S_{r}, f(S)_{r}$, and $\operatorname{Gr}\left(f \uparrow_{S}\right)_{r}$ constitute the filtration $\left\{S_{r} \leftarrow \operatorname{Gr}\left(f \uparrow_{S}\right)_{r} \rightarrow f(S)_{r}\right\}$ in $\operatorname{Top}^{(\mathrm{bf})}$ with morphisms induced by inclusions such that the diagram

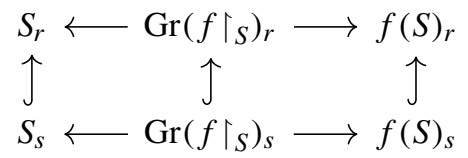

commutes for every $s \leq r \in \mathbb{R}_{\geq 0}$.

In the same way as that for the discrete analysis, applying homology functor $H$ to the filtration produces $\left\{H S_{r} \leftarrow H \mathrm{Gr}\left(f\left\lceil_{S}\right)_{r} \rightarrow H f(S)_{r}\right\}\right.$, which is a family of objects in the representation category $\operatorname{rep}\left(A_{3}(b f)\right)$ with induced morphisms from Diagram (5).

Remark 5.4 We have constructed different representations from earlier representations using complexes in Sect. 4.1, but these are isomorphic if we adopt Čech complexes. By the Nerve Lemma (Borsuk 1948), it is known that if $U$ is a finite subset in a metric space, then the sublevel set $U_{r}$ is homotopy equivalent to the Čech complex of $U$ with radius $r$. Therefore, letting $C_{r}, G_{r}$, and $D_{r}$ be Čech complexes with radius $r$ of the finite subsets $S, \operatorname{Gr}\left(f \uparrow_{S}\right)$, and $f(S)$, respectively, the induced family $\left\{H C_{r} \leftarrow H G_{r} \rightarrow H D_{r}\right\}$ is isomorphic to the family $\left\{H S_{r} \leftarrow H \mathrm{Gr}\left(f \uparrow_{S}\right)_{r} \rightarrow H f(S)_{r}\right\}$.

Because decomposing every representation into intervals is isomorphic in the functor category $\operatorname{rep}\left(A_{3}(b f)\right)^{\mathbb{R}}$, the family $\left\{H S_{r} \leftarrow H \mathrm{Gr}\left(f \uparrow_{S}\right)_{r} \rightarrow H f(S)_{r}\right\}$ is isomorphic to $\left\{\bigoplus_{1 \leq b \leq d \leq 3} \mathbb{I}[b, d]^{m_{b, d}^{r}}\right\}$. The induced morphisms can be written in block matrix form again.

Lemmas 4.4 and 4.6 uniquely determine the family $\left\{\mathbb{I}[1,3]^{m_{1,3}^{r}}\right\}$ and the induced morphisms up to isomorphism. The family and the morphisms give us three copies of the $\mathbb{R}$-persistence module $\left\{K^{m_{1,3}^{r}}=K^{m_{1,3}^{r}}=K^{m_{1,3}^{r}}\right\}$. Therefore, we obtain an $\mathbb{R}$-persistence module $\left\{K^{m_{1,3}^{r}}\right\}$. We denote this $\mathbb{R}$-persistence module of the sampled map $f \uparrow_{S}$ as $M^{f \uparrow_{S}}$ and call it the $\mathbb{R}$-persistence module of the sampled map.

Remark 5.5 The construction of an $\mathbb{R}$-persistence module using graphs requires no assumption that $S$ is a finite set. Therefore, if we assume that $\operatorname{dim} H X_{r}, \operatorname{dim} H \operatorname{Gr}(f)_{r}$, and $\operatorname{dim} H f(X)_{r}$ are finite for every $r$, then we can execute the same analysis of the filtration $\left\{X_{r} \leftarrow \operatorname{Gr}(f)_{r} \rightarrow f(X)_{r}\right\}$, deriving an $\mathbb{R}$-persistence module $M^{f}$ in the 
same way. We designate this as the $\mathbb{R}$-persistence module of the map $f$. The output persistence diagram portrays the robustness of the generators of the homology-induced $\operatorname{map} f_{*}$.

After this setup, we can show the following stability theorem.

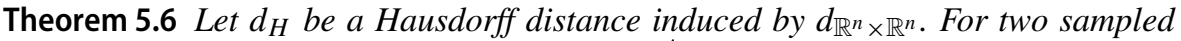
maps $h: S \rightarrow \mathbb{R}^{n}$ and $h^{\prime}: S^{\prime} \rightarrow \mathbb{R}^{n}$, let $M^{h}, M^{h^{\prime}}$ be the $\mathbb{R}$-persistence modules of the sampled maps. Then,

$$
d_{I}\left(M^{h}, M^{h^{\prime}}\right) \leq d_{H}\left(\operatorname{Gr}(h), \operatorname{Gr}\left(h^{\prime}\right)\right) .
$$

Proof Let $\varepsilon:=d_{H}\left(\operatorname{Gr}(h), \operatorname{Gr}\left(h^{\prime}\right)\right)$. Also, let $r$ be an arbitrary non-negative real number.

By the definition of Hausdorff distance, $\operatorname{Gr}(h)_{r} \subset \operatorname{Gr}\left(h^{\prime}\right)_{r+\varepsilon}$ and $\operatorname{Gr}\left(h^{\prime}\right)_{r} \subset$ $\operatorname{Gr}(h)_{r+\varepsilon}$. Moreover, $\varepsilon=d_{H}\left(\operatorname{Gr}(h), \operatorname{Gr}\left(h^{\prime}\right)\right)$ implies that $d_{H}\left(S, S^{\prime}\right) \leq \varepsilon$ and $d_{H}\left(h(S), h^{\prime}\left(S^{\prime}\right)\right) \leq \varepsilon$. Hence $S_{r} \subset S_{r+\varepsilon}^{\prime}, S_{r}^{\prime} \subset S_{r+\varepsilon}, h(S)_{r} \subset h^{\prime}\left(S^{\prime}\right)_{r+\varepsilon}$, and $h^{\prime}\left(S^{\prime}\right)_{r} \subset h(S)_{r+\varepsilon}$ as well. These inclusions engender the following commutative diagrams:

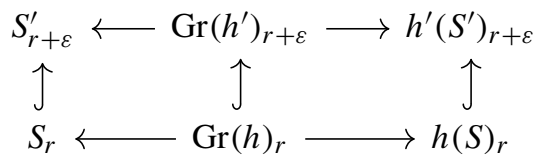

and

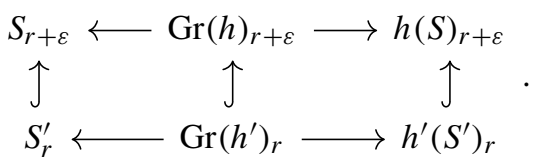

By that functoriality, it is straightforward that these inclusions induce $\varepsilon$-interleaving morphisms between $M^{h}$ and $M^{h^{\prime}}$. Then we have $d_{I}\left(M^{h}, M^{h^{\prime}}\right) \leq \varepsilon$.

Accordingly, the obtained persistence modules and persistence diagrams can only have as much noise as $S$ or its evaluation by $f$.

The proof uses no the assumption that $S$ is a finite set. Therefore, a similar inequality holds for $\mathbb{R}$-persistence modules of maps.

Corollary 5.7 Let $U$ and $U^{\prime}$ be subsets in $\mathbb{R}^{n}$. If $\mathbb{R}$-persistence modules $M^{h}$ and $M^{h^{\prime}}$ of maps $h: U \rightarrow \mathbb{R}^{n}$ and $h^{\prime}: U^{\prime} \rightarrow \mathbb{R}^{n}$ are defined, then

$$
d_{I}\left(M^{h}, M^{h^{\prime}}\right) \leq d_{H}\left(\operatorname{Gr}(h), \operatorname{Gr}\left(h^{\prime}\right)\right) .
$$

By Corollary 5.7, the error (bottleneck distance) between the persistence diagram of a sampled map $f\left\lceil_{S}\right.$ and that of the original map $f$ is bounded above by the error $d_{H}\left(\operatorname{Gr}(f), \operatorname{Gr}\left(\left.f\right|_{S}\right)\right)$ of the sampled map. If the sampled map is sufficiently dense, then we can infer the persistent generators of $f_{*}$ from the persistence module of the sampled map. 
Finally, let us provide the stability of the persistence analysis using grids. We regard the persistent homology constructed using a grid as an $\mathbb{R}$-persistence module by the embedding induced by

$$
F_{r}:=\left\{\begin{array}{ll}
\emptyset & (r<\varepsilon) \\
F_{i \varepsilon} & \left(i \varepsilon \leq r<(i+1) \varepsilon \text { for } i \in \mathbb{Z}_{\geq 1}\right)
\end{array} .\right.
$$

Theorem 5.8 Let $d_{H}$ be a Hausdorff distance induced by $d_{\infty}$. For two sampled maps $h: S \rightarrow \mathbb{R}^{n}$ and $h^{\prime}: S^{\prime} \rightarrow \mathbb{R}^{n}$, we write the filtrations of correspondences as $\left\{F_{r}\right\}$ and $\left\{F_{r}^{\prime}\right\}$ and let $M^{h}$ and $M^{h^{\prime}}$ be their output $\mathbb{R}$-persistence modules. If $d_{H}\left(\operatorname{Gr}(h), \operatorname{Gr}\left(h^{\prime}\right)\right) \leq \varepsilon$, then $d_{I}\left(M^{h}, M^{h^{\prime}}\right) \leq \varepsilon$.

Proof The assumption $d_{H}\left(\operatorname{Gr}(h), \operatorname{Gr}\left(h^{\prime}\right)\right) \leq \varepsilon$ derives the inequality $d_{H}\left(F_{\varepsilon}^{h}, F_{\varepsilon}^{h^{\prime}}\right) \leq$ $\varepsilon$. Therefore, there exist the following inclusions of

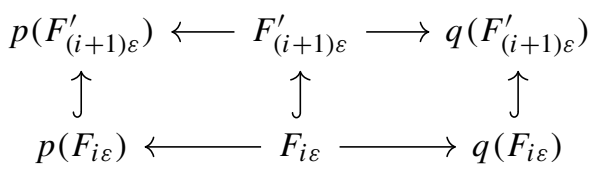

and

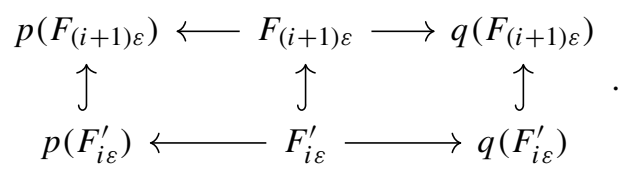

It might be readily apparent that these inclusions induce the $\varepsilon$-interleaving morphisms between $M^{h}$ and $M^{h^{\prime}}$.

\section{Application of functoriality to 2-D persistence modules}

The functoriality lemma, Lemma 4.4, can be generalized for the restriction to every "diagonal" block. Precisely because the candidate of intervals $\mathbb{I}[c, d]$ satisfying relations $\mathbb{I}[a, b] \unrhd \mathbb{I}[c, d] \unrhd \mathbb{I}[a, b]$ is only $\mathbb{I}[c, d]=\mathbb{I}[a, b]$,

$$
[\Psi \Theta]_{a: b}^{a: b}=\Psi_{a: b}^{a: b} \Theta_{a: b}^{a: b}
$$

holds for all $\mathbb{I}[a, b]$. This result can be checked, not only on the orientation $b f$ but also on every orientation of any length, as described below.

Presuming that $\mathbb{I}[c, d] \neq \mathbb{I}[a, b]$, which can happen when $a \neq c$ or when $b \neq d$. In the case in which $a \neq c$, one might assume $a<c$ without loss of generality. When $(c-1)$-th orientation is $f$, consider $g=\left\{g_{i}\right\}_{i=1}^{n} \in \operatorname{Hom}(\mathbb{I}[a, b], \mathbb{I}[c, d])$. Then, the commutative diagram of the morphism $g$ from $(c-1)$ to $c$ is 


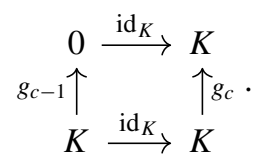

It is readily apparent that $g_{c-1}=0$. The commutativity derives $g_{c}=0$. Because the commutativity of the diagram on $g$ derives $g_{i}=0$ for the other vertices $i, g=0$. Consequently, $\operatorname{Hom}(\mathbb{I}[a, b], \mathbb{I}[c, d])=0$. Therefore, $\mathbb{I}[a, b] \unrhd \mathbb{I}[c, d]$. We can show $\mathbb{I}[c, d] \not \mathbb{I}[a, b]$ when $(c-1)$-th orientation is $b$ in a similar discussion, using the commutative diagram as

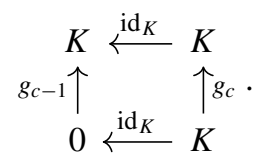

Similar arguments also hold in the case in which $b \neq d$, concluding $\mathbb{I}[a, b] \not \mathbb{I}[c, d]$ or $\mathbb{I}[c, d] \unrhd \mathbb{I}[a, b]$.

Consequently, we can extend the statement on the orientation $b f$ to general $\tau_{n}$ as shown below.

\section{Proposition 6.1 Let}

$$
\Theta: \bigoplus_{1 \leq a \leq b \leq n} \mathbb{I}[a, b]^{m_{a, b}^{1}} \rightarrow \bigoplus_{1 \leq a \leq b \leq n} \mathbb{I}[a, b]^{m_{a, b}^{2}}
$$

and

$$
\Psi: \bigoplus_{1 \leq a \leq b \leq n} \mathbb{I}[a, b]^{m_{a, b}^{2}} \rightarrow \bigoplus_{1 \leq a \leq b \leq n} \mathbb{I}[a, b]^{m_{a, b}^{3}}
$$

be block matrix forms of objects in the arrow category $\operatorname{arr}\left(\operatorname{rep}\left(A_{n}\left(\tau_{n}\right)\right)\right)$. Then

$$
[\Psi \Theta]_{a: b}^{a: b}=\Psi_{a: b}^{a: b} \Theta_{a: b}^{a: b}
$$

for all $1 \leq a \leq b \leq n$.

We can use this property for 2-D persistence modules, which are representations with the shape of

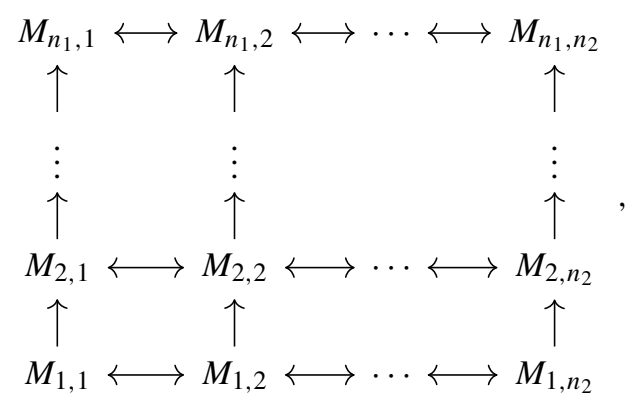


where every row has the same orientation $\tau_{n_{2}}$. The 2-D persistence modules sometimes appear and create difficulties in the context of persistence analysis for time series data. Carlsson and Zomorodian (2009) provide details and higher dimensional persistence.

In our context, the 2-D persistence module naturally appears when we consider iterations of a sampled map or compositions of sampled maps. Presuming that we have a time series of some point clouds $\left\{S_{1}, S_{2}, \ldots, S_{T}\right\}$ in the same Euclidean space, with their transition as maps $\left\{f_{i}: S_{i} \rightarrow S_{i+1}\right\}$, then we generate a filtration of abstract simplicial complexes $C_{1}^{t} \subset \cdots \subset C_{n}^{t}$ for each $S_{t}$. As shown in Sect. 4, the maps between points induce a filtration of partial maps $f_{i}^{t}: C_{i}^{t} \nrightarrow C_{i}^{t+1}$, which induces a commutative diagram as

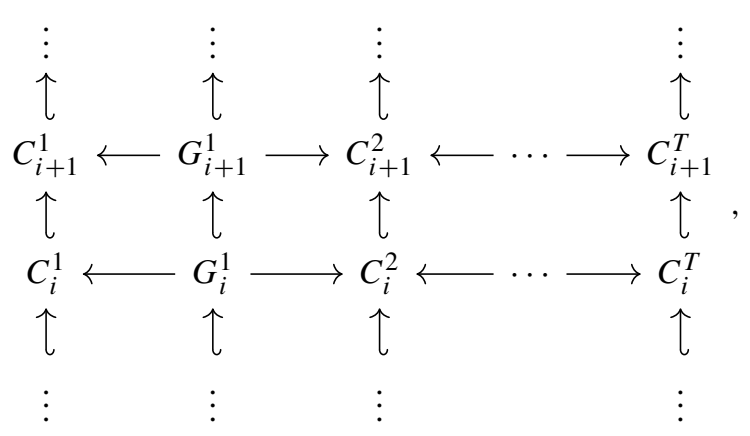

by taking the $i$-th simplicial complex $G_{i}^{t}$ of $\operatorname{Gr}\left(f_{i}^{t}\right)$ defined as Equation (1). As a consequence, the homology functor induces the 2-D persistence module from the diagram above. In this case, we can observe the 2-D persistence module from the viewpoint that the horizontal (vertical) direction on the diagram describes persistence in time (space, respectively).

Diagram (6) can be reviewed. In the same way as in the specific case $\tau_{n_{2}}=b f$, Diagram (6) can be regarded as a sequence of morphisms in the category $\operatorname{rep}\left(A_{n_{2}}\left(\tau_{n_{2}}\right)\right)$. By decomposing representations of $A_{n_{2}}\left(\tau_{n_{2}}\right)$ in each row, the morphisms can be dealt with as matrices in block matrix form. Restricting each matrix to the diagonal block $(a: b, a: b)$ derives a sequence of matrices for which the domains and codomains are direct sums of $\mathbb{I}[a, b]$. Because this sequence comprises $b-a$ copies of nonzero representations of $A_{n_{1}}$ and $n_{2}-(b-a)$ copies of zero representations, one of the nonzero representations can be selected. Finally, we obtain the persistence diagram by decomposition. Proposition 6.1 ensures the uniqueness of the output persistence diagram.

For 2-D persistence modules derived from Diagram (7), when we take the block $(a: b, a: b)$ as $\left(1: n_{2}, 1: n_{2}\right)$, each generator of the output persistence module survives under all transitions. Its lifetime in the persistence diagram shows how robust it is in the Euclidean space. Although this process ignores much information stored in the other blocks, it is an approach to 2-D persistence analysis that is able to capture the rough topological structures. 


\section{Numerical experiments}

The author has implemented the persistence analysis introduced into Sect. 4.1. The implementation is available at the GitHub repository https://github.com/hiroshitakeuchi/ pdsm. Before presenting some numerical results, we outline the implementation strategy. Here we fix the field for the coefficient of matrices and the homology functor as $\mathbb{Z} / 1009 \mathbb{Z}$.

Remark 7.1 To implement the persistence analysis of computers, we must use finite fields as the coefficient. Here, every map is written as a matrix. If we choose field $\mathbb{Z} / 2 \mathbb{Z}$ as the coefficient, then every entry with the prime factor 2 in the matrix is regarded as 0 . For example, a homology generator $a$ mapped as $f_{*}(a)=2 a$, such as the example discussed later and in Fig. 4, is ignored. Therefore, it is better to choose a larger prime number $p$ as the coefficient for $\mathbb{Z} / p \mathbb{Z}$ to retrieve more generators.

The implementation uses Vietoris-Rips complexes for simplicity, whereas Čech complexes are theoretically more satisfying (see Edelsbrunner and Harer (2010, Section III.2) for details). Except for construction of the persistence module from the sequence of the pairs of the maps $\left\{\left(p_{i_{*}}, q_{i *}\right)\right\}$, it fundamentally follows the algorithm in Edelsbrunner et al. (2015) (recall Remark 4.3).

First, we generate the boundary matrix induced by the filtration of the Vietoris-Rips complexes for each point cloud $S$ and $f(S)$; then the boundary matrix of the filtration $\left\{G_{i}\right\}$. We can use the original persistence algorithm (Edelsbrunner and Harer 2010) to compute the reduced boundary matrices and the bases of the persistent homology of the filtration.

Second, because we can obtain the homology bases for each filtration, we generate the maps $p_{i_{*}}$ and $q_{i_{*}}$ as matrices between the homology basis for each filter. Similarly, we compute the induced maps of the inclusions $j_{*}: H G_{i} \rightarrow H G_{i+1}$ as matrices. To obtain the basis of $\mathbb{I}[1,3]$ for each matrix $p_{i_{*}}$ and $q_{i_{*}}$, we execute the following elementary row and column operations.

1. Transform $p_{i *}$ to Smith normal form as

$$
P_{1} p_{i_{*}} Q_{1}=\left[\begin{array}{cc}
I_{r_{1}} & 0 \\
0 & 0
\end{array}\right],
$$

where $P_{1}$ and $Q_{1}$ are regular matrices corresponding to elementary operations, and where $r_{1}$ is the rank of $p_{i *}$.

2. Because $p_{i_{*}}$ and $q_{i *}$ share the same basis for the columns, the elementary column operations $Q_{1}$ are performed simultaneously on $q_{i *}$

$$
q_{i *} Q_{1}=\left[\begin{array}{ll}
X_{1} & X_{2}
\end{array}\right]
$$

where $X_{1}$ is the submatrix based on columns corresponding to the above $I_{r_{1}}$, and $X_{2}$ is the submatrix corresponding to the 0 columns in $P_{1} p_{i_{*}} Q_{1}$. 
3. Transform $X_{2}$ to Smith normal form with elementary row operations $P_{2}$ and elementary column operations $Q_{2}$ as

$$
\left[\begin{array}{ccc}
P_{2} X_{1} & I_{r_{2}} & 0 \\
& 0 & 0
\end{array}\right]=\left[\begin{array}{ccc}
X_{3} & I_{r_{2}} & 0 \\
X_{4} & 0 & 0
\end{array}\right]
$$

where $P_{2} X_{1}$ is divided into submatrices of appropriate sizes on the right-hand side. We remark that these column operations have no side effect on the $p_{i *}$ side matrix because every column corresponding to the basis is zero.

4. Zero out $X_{3}$ by $I_{r_{2}}$ using column operations with no side effect as

$$
\left[\begin{array}{ccc}
0 & I_{r_{2}} & 0 \\
X_{4} & 0 & 0
\end{array}\right]
$$

5. Transform $X_{4}$ to Smith normal form with elementary row operations $P_{4}$ and elementary column operations $Q_{4}$ as

$$
\left[\begin{array}{cccc}
0 & I_{r_{2}} & 0 \\
I_{r_{3}} & 0 & 0 & 0 \\
0 & 0 & & 0
\end{array}\right]
$$

Here the column operations have side effect on $I_{r_{1}}$ transforming it to a matrix $Q_{4}$, but $Q_{4}$ is regular. Therefore, we can transform it to $I_{r_{1}}$ again using only row operations.

6. Finally, we obtain the matrix transformations as

$$
p_{i_{*}} \mapsto\left[\begin{array}{cccc}
I_{r_{3}} & 0 & 0 & 0 \\
0 & I_{r_{3}-r_{1}} & 0 & 0
\end{array}\right] \text { and } q_{i_{*}} \mapsto\left[\begin{array}{cccc}
0 & 0 & I_{r_{2}} & 0 \\
I_{r_{3}} & 0 & 0 & 0 \\
0 & 0 & 0 & 0
\end{array}\right]
$$

which are decomposed into intervals. The rows and columns corresponding to two $I_{r_{3}}$ are pairs of identity maps, which are $\mathbb{I}[1,3]$. Therefore the basis in the columns of $I_{r_{3}}$ is what we want.

By applying the change of basis of $H G_{i}$ during the above column operations and restricting to the basis corresponding to $I_{r_{3}}$, one can finally obtain the persistent homology of the sampled map. Finally, after decomposing the persistent homology into intervals using the decomposition algorithm in Edelsbrunner et al. (2015, Subsection 3.4), a persistence diagram can be plotted.

By tracking the inverse of the matrix transformations executed above, one can write down the cycles corresponding to the generators in the persistence diagram. As remarked in Sect. 3, the cycles depend on the choice of the bases of $\mathbb{I}[1,3]$. Nevertheless, for the following numerical experiments, we succeed in reconstructing the underlying maps. To emphasize a contrast, we present examples of failed reconstructions in Sect. 8. 


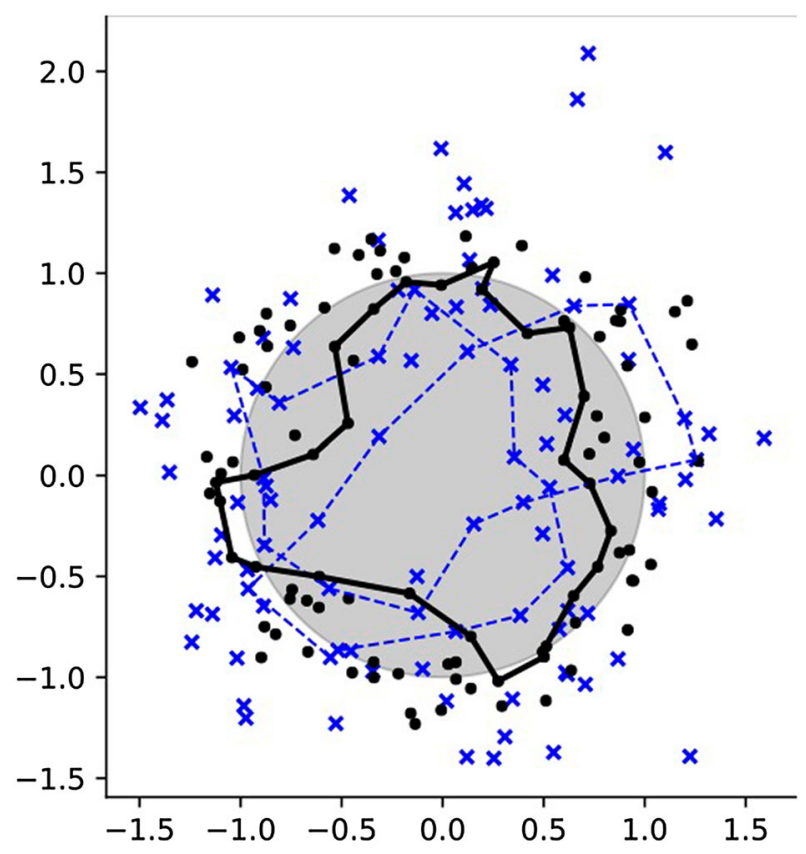

Fig. 4 Computational result for $f(z)=z^{2}$. The number of points is 100 . The Gaussian noise is at $\sigma=0.18$. The black points are sampled points for the domain. The blue crosses are its image by $f$. As presented in Fig. 5, the generator is unique. The corresponding generator in the domain side is described by the black edges approximating the unit circle. The generator in the image side is the blue dashed edges. One can observe that it turns around the origin twice

\subsection{Twice mapping on a circle}

As an example of input data, one can consider the twice map on the unit circle $f: S^{1} \rightarrow$ $S^{1}$ defined as $f(z):=z^{2}$. It is noteworthy that, in this case, the spaces in Problem 4.1 are given as $X=Y=S^{1}$ embedded in $\mathbb{R}^{2}$. Then we regard $\mathbb{R}^{2}$ as $\mathbb{C}$. The sampled points of the unit circle are 100 points $z_{j}:=\cos \left(2 \pi \frac{j}{100}\right)+\sqrt{-1} \sin \left(2 \pi \frac{j}{100}\right)$ for $0 \leq j<100$, with added Gaussian noise with $\sigma \in[0.00,0.30]$.

A computational result is presented in Fig. 4, which portrays the sampled map at $\sigma=0.18$ and its unique generator of the persistence diagram. The generator is the corresponding cycle in $H G_{b}$ at the birth radius $b$. It is indeed approximating the unit circle. Then it is apparent that its image is turning around the origin twice. The results for other noises are presented in Fig. 6.

Figure 5 presents the persistence diagrams under changing $\sigma$ from 0 to 0.3 . As expected, the lifetime of the unique generator decreases as the noise increases.

\subsection{Inverse mapping on a circle}

To emphasize the difference from the existing method using eigenspace functors, one can consider the inverse map on the unit circle $g: S^{1} \rightarrow S^{1}$ defined as $f(z):=z^{-1}$. 

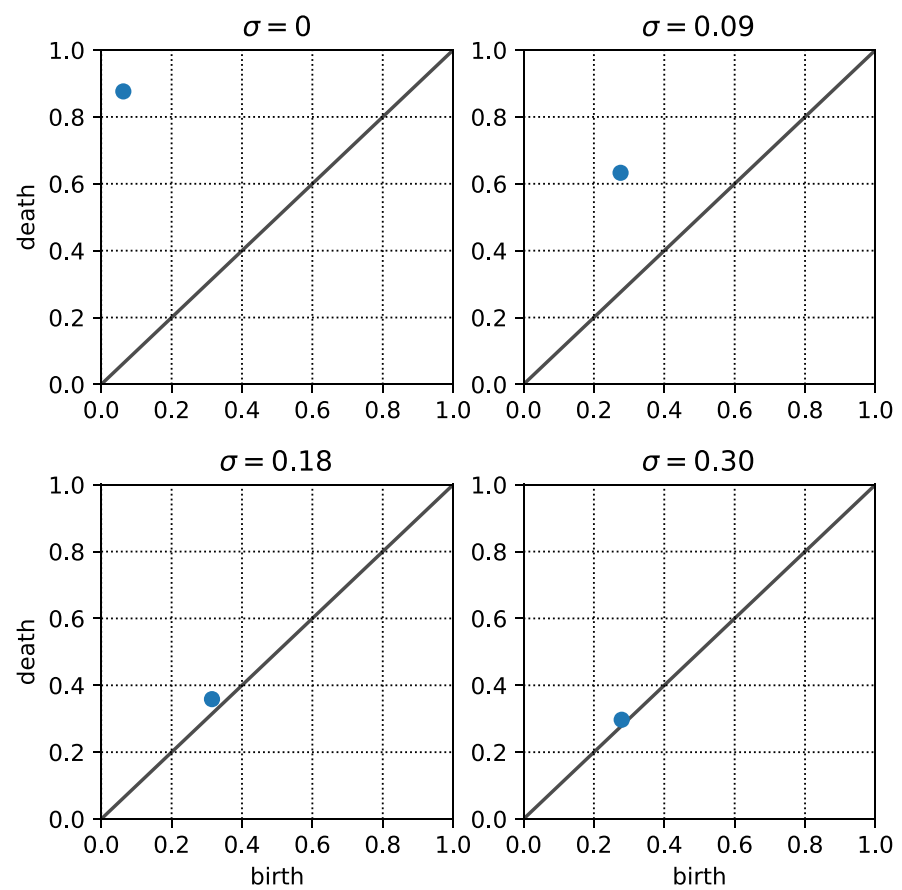

Fig. 5 Persistence diagrams for $f(z)=z^{2}$ with 100 points at $\sigma=0.00,0.09,0.18$, and 0.30 . We can observe that each persistence diagram has the unique point. It approaches the diagonal line as the noise increases

The sampled points $\left\{z_{j}\right\}$ and the range of the parameter $\sigma$ of Gaussian noises are the same as before. The computational results are presented in Figs. 7 and 8.

The analysis using eigenspace functors can detect such a generator using the eigenspace functor with eigenvalue -1 . In other words, prior knowledge about the eigenvalue is fundamentally important. By contrast, our method can use the same construction both for the inverse mapping and twice mapping.

\subsection{Mapping on a torus}

Moreover, our method is applicable to maps on the torus $T:=\mathbb{R}^{2} / \mathbb{Z}^{2}$. We adopt the metric on $T$ induced by the Euclidean metric on $\mathbb{R}^{2}$.

One can consider the self-map on $T$ defined as

$$
A=\left(\begin{array}{ll}
2 & 1 \\
1 & 1
\end{array}\right): T \rightarrow T
$$

We set 64 sampling points $S:=\left\{\left(\frac{i}{8}, \frac{j}{8}\right) \mid 0 \leq i \leq 7,0 \leq j \leq 7\right\}$ and generate a sampled map of $A$ on $S$. Such a sampled map of $A$ is a challenging example for 

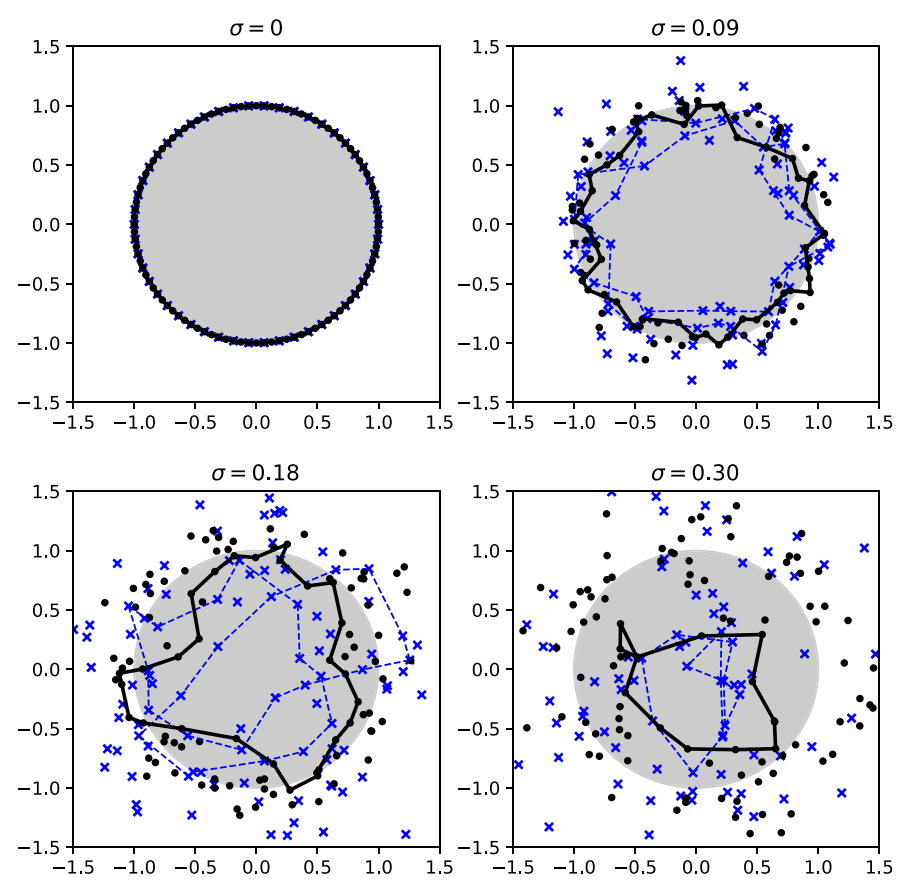

Fig. 6 Computational results obtained for $f(z)=z^{2}$ with 100 points at $\sigma=0.00,0.09,0.18$, and 0.30 . Every plotting range is restricted to $[-1.5,1.5] \times[-1.5,1.5]$ to observe the generators. The corresponding persistence diagrams are in Fig. 5

analysis using eigenspace with no prior knowledge because the eigenvalues of $A$ are $\frac{3+\sqrt{5}}{2}$ and $\frac{3-\sqrt{5}}{2}$.

The computational results are presented in Figs. 9, 10, 11, 12, 13, 14 and 15. We remark that the unique point in Fig. 9 has multiplicity 2. Let $\left(\begin{array}{l}1 \\ 0\end{array}\right)$ and $\left(\begin{array}{l}0 \\ 1\end{array}\right)$ be a standard homology basis on the torus. The generators corresponding to the unique birth-death point are given as $\alpha$ and $505 \alpha+\beta\left(=\frac{1}{2} \alpha+\beta\right.$ with the coefficients in $\left.\mathbb{Z} / 1009 \mathbb{Z}\right)$ in our numerical experiment, where $\alpha$ and $\beta$ respectively denote cycles in $H G_{b}$ at the birth radius $b$ illustrated in Figs. 10, 11, 12, 13, 14 and 15. These cycles correspond to the mappings

$$
\left(\begin{array}{c}
-1 \\
0
\end{array}\right) \mapsto\left(\begin{array}{c}
-2 \\
-1
\end{array}\right) \text { and }\left(\begin{array}{l}
0 \\
1
\end{array}\right) \mapsto\left(\begin{array}{l}
1 \\
1
\end{array}\right)
$$

respectively. The mappings (8) are nothing but the map $A=\left(\begin{array}{ll}2 & 1 \\ 1 & 1\end{array}\right)$, concluding the success in reconstructing $A$. 

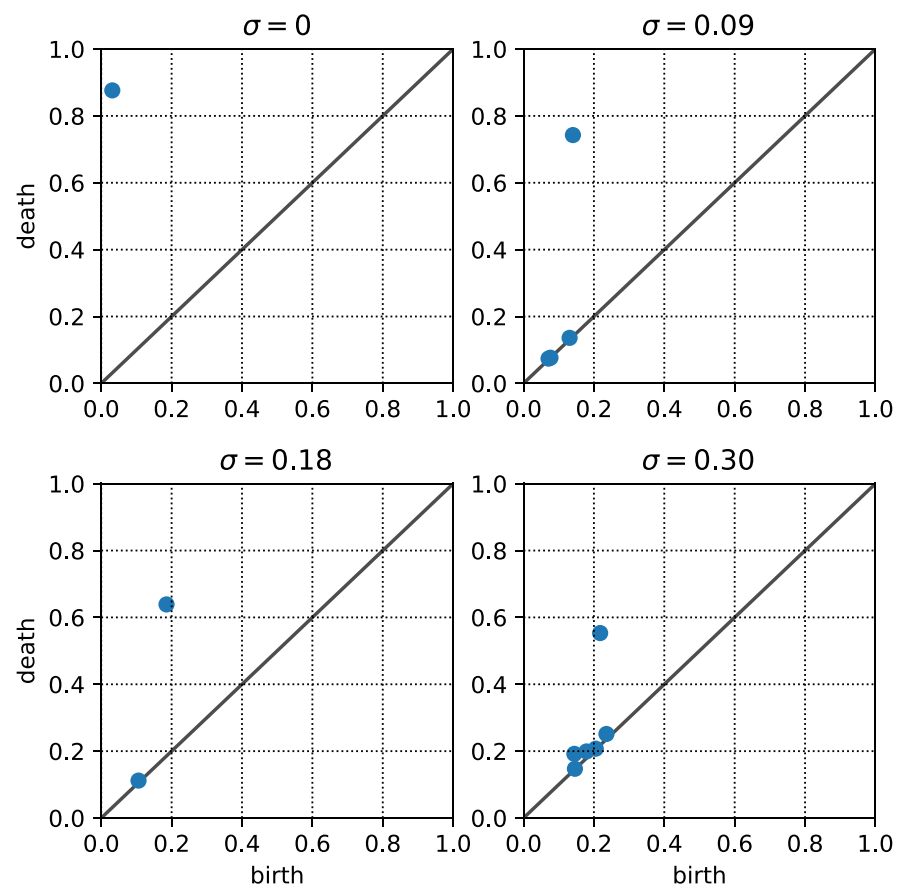

Fig. 7 Persistence diagrams for $f(z)=z^{-1}$ with 100 points at $\sigma=0.00,0.09,0.18$, and 0.30

\section{Examples of failed reconstructions}

In the preceding section, we performed reconstruction of each map at the filter where the generator was born. However, such reconstruction is not always possible. This section presents two examples of failed reconstructions.

\subsection{Non-optimal example}

The first example appears even in ordinary homology groups. The example is known as optimal cycles of homology groups, as described by Escolar and Hiraoka (2014).

We consider the simplicial complex $C_{1}$ presented in Fig. 16 and the identity map $\operatorname{id}_{C_{1}}$ on $C_{1}$ by taking three copies of $C_{1}$ as

$$
C_{1} \stackrel{=}{=} C_{1} \stackrel{=}{\longrightarrow} C_{1} .
$$

We remark that the simplicial complex of the graph $\operatorname{Gr}\left(\mathrm{id}_{C_{1}}\right)$ of the identity map on $C_{1}$ is isomorphic to $C_{1}$ by definition introduced as (1). We therefore regard (9) as a filtration consisting of only one filter.

Taking the 1-dimensional homology $H=H_{1}(-; K)$, we have the representation

$$
H C_{1} \stackrel{=}{\longleftarrow} H C_{1} \stackrel{=}{\longrightarrow} H C_{1}
$$



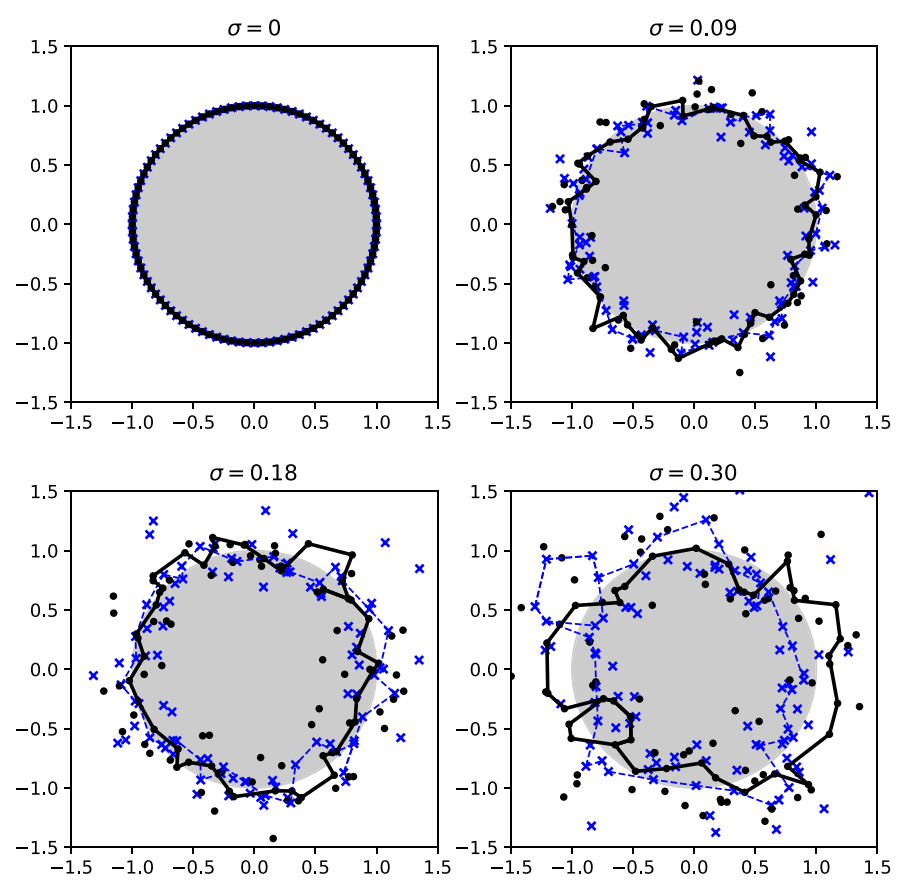

Fig. 8 Computational results for $f(z)=z^{-1}$ with 100 points at $\sigma=0.00,0.09,0.18$, and 0.30 . The generators correspond to the most persistent birth-death pair in each persistence diagram in Fig. 7

Fig. 9 Persistence diagram of a

sampled map of $A=\left(\begin{array}{ll}2 & 1 \\ 1 & 1\end{array}\right)$.

The unique point has

multiplicity 2

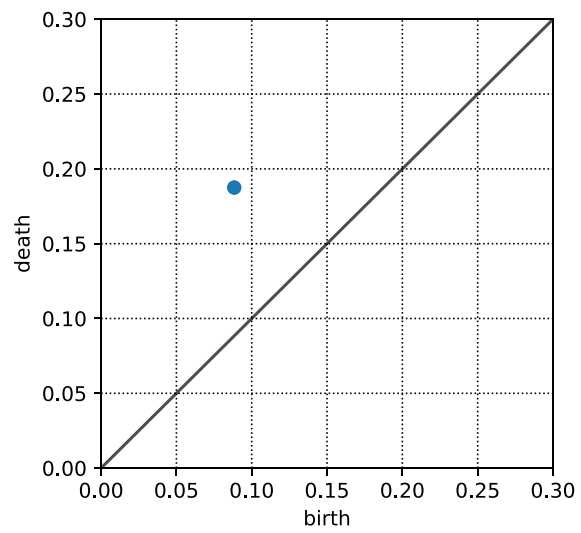

isomorphic to

$$
K^{2} \stackrel{=}{\longleftarrow} K^{2} \stackrel{=}{\longrightarrow} K^{2}
$$

that is two copies of the interval $\mathbb{I}[1,3]$. To obtain the representation (11), some isomorphism must be taken from (10) to (11). The problem for reconstruction is how to choose cycles as the basis of the homology groups. One option is to choose cycles $\alpha$ and $\gamma$. However, this is surplus because choosing $\alpha$ and $\beta$ is more straightforward for the number of simplices used. It is natural for the reconstruction to use $\alpha$ and $\beta$. 
Fig. 10 Homology generator $\alpha$ on the torus corresponding to the point in Fig. 9. The black line is the cycle in domain and mapped to the blue dashed line. Using the standard basis, this mapping is written as $\left(\begin{array}{c}-1 \\ 0\end{array}\right) \mapsto\left(\begin{array}{l}-2 \\ -1\end{array}\right)$

Fig. 11 Generator on the domain side embedded in $\mathbb{R}^{3}$. The loop turns around the torus once in a longitudinal direction

Fig. 12 Generator on the image side embedded in $\mathbb{R}^{3}$. The loop turns around the torus twice in a longitudinal direction and once in a meridian direction

Fig. 13 Another generator $\beta$ showing $\left(\begin{array}{l}0 \\ 1\end{array}\right) \mapsto\left(\begin{array}{l}1 \\ 1\end{array}\right)$. In our numerical experiment, the other generator corresponding to the point in Fig. 9 is given as $505 \alpha+\beta\left(=\frac{1}{2} \alpha+\beta\right.$ with the coefficients in $\mathbb{Z} / 1009 \mathbb{Z}$ )
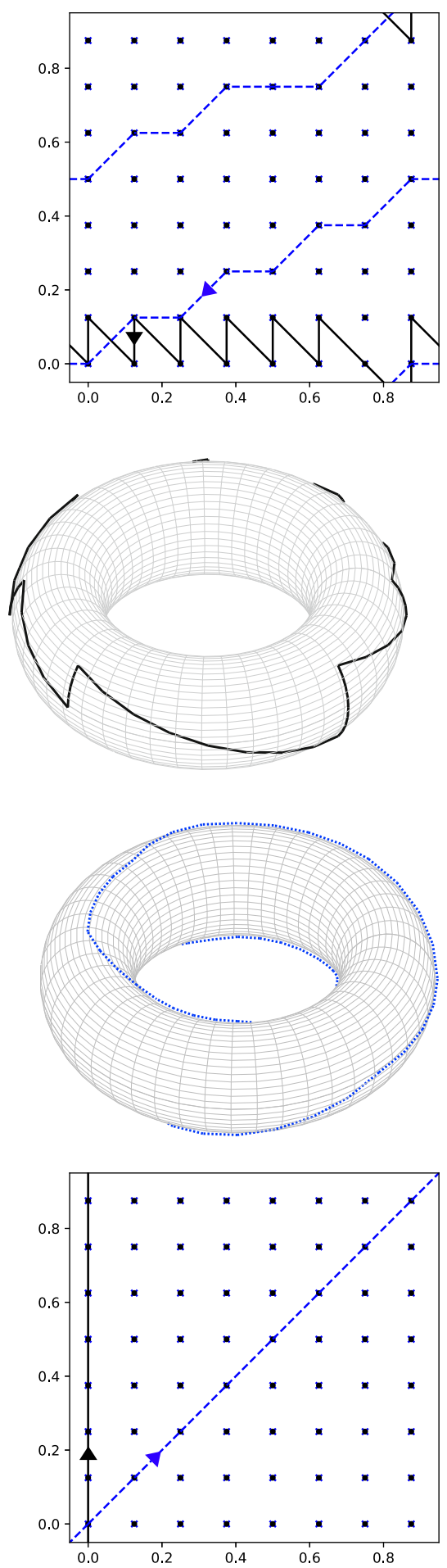
Fig. 14 Generator on the domain side embedded in $\mathbb{R}^{3}$.

The loop turns around the torus once in a meridional direction

Fig. 15 Generator on the image side embedded in $\mathbb{R}^{3}$. The loop turns around the torus once in a longitudinal and once in a meridional direction
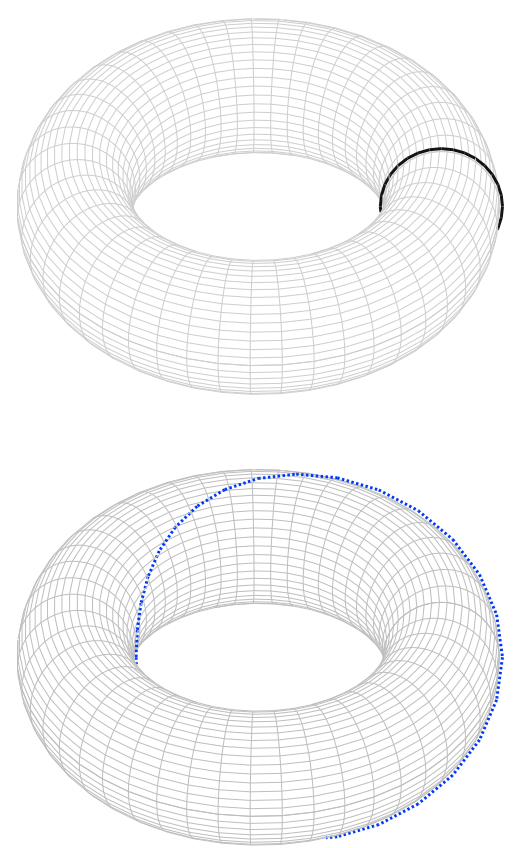

Fig. 16 Simplicial complex $C_{1}$ with the 1 -cycles $\alpha, \beta$, and $\gamma$. The outer 1-cycle $\gamma$ is homologous to $\alpha+\beta$

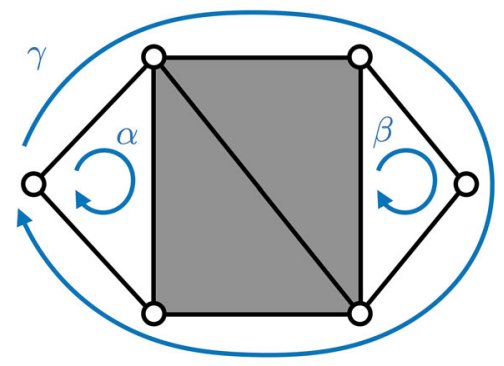

Another example of non-optimal cycles is shown in Fig. 8. The cycle at $\sigma=$ 0.30 consists of a larger cycle and a smaller one. It is sufficient and better for the reconstruction of the unit circle to take only the larger cycle.

For the case in which we have one complex, one can solve such an optimization problem using optimal cycles (Escolar and Hiraoka 2014). Furthermore, for the case in which one has a filtration of simplicial complexes, one can solve the optimization problem using the persistent homology version of optimal cycles (Obayashi 2018). Nevertheless, no general optimization method is applicable to $A_{3}(b f)$ type representations.

\subsection{Inconclusive example}

We now consider the simplicial complexes depicted in Fig. 17. 


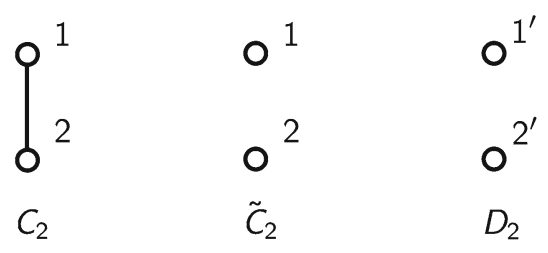

Fig. 17 Left and middle: a simplicial complex $C_{2}$ consisting of the two points 1 and 2 connected with an edge, and its subcomplex $\tilde{C}_{2}$ without edge. Right: a simplicial complex $D_{2}$ consisting of the two points $1^{\prime}$ and $2^{\prime}$ without edge

Defining the sampled map as $1 \mapsto 1^{\prime}$ and $2 \mapsto 2^{\prime}$, the simplicial complex of the graph of the sampled map is isomorphic to $\tilde{C}_{2}$. Similarly to Sect. 8.1, we regard

$$
C_{2} \longleftrightarrow \tilde{C}_{2} \longrightarrow D_{2}
$$

as a filtration consisting of only one filter, where the left arrow is the inclusion map and the right is induced by the sampled map.

Taking the 0 -dimensional homology $H=H_{0}(-; K)$, we obtain the representation of

$$
H C_{2} \longleftarrow H \tilde{C}_{2} \longrightarrow H D_{2}
$$

isomorphic to

$$
K \longleftarrow K^{2} \stackrel{=}{\longrightarrow} K^{2}
$$

that is the direct sum of $\mathbb{I}[1,3]$ and $\mathbb{I}[2,3]$. To obtain the representation (13), some isomorphism must be taken from (12) to (13). The problem again is how to choose cycles as the basis of the homology groups. In this case, we have the two simplest options. One is to choose 1 for $C_{2}$ and $\tilde{C}_{2}$ and $1^{\prime}$ for $D_{2}$. The other is to choose 2 for $C_{2}$ and $\tilde{C}_{2}$ and $2^{\prime}$ for $D_{2}$. We remark that 1 is homologous to 2 in $C_{2}$. Then they form the unique connected component. The image of the component depends on the choice of 1 or 2 in $\tilde{C}_{2}$ for the corresponding basis. However, we have no information to decide which side is better to choose. Consequently, all that can be said here is that the unique connected component in $C_{2}$ is still non-trivial in $D_{2}$ after the mapping in this filter.

In summary, each point of the persistence diagram of a sampled map implies non-trivial homology generators of the domain and codomain at the filter where the corresponding generator is born. The homology of the simplicial complex of the graph connects the generators, which are as robust as the lifetime in the persistent homology. In other words, the filter possesses non-trivial and robust topological information of the sampled map. Therefore, as presented in the examples of the preceding section and in this one, exploring the filter $H C_{b} \leftarrow H G_{b} \rightarrow H D_{b}$ at birth $b$ will help elucidate the topological transition under the sampled map, even for the case of failed reconstructions. 


\section{Concluding remarks}

As described herein, we defined the persistence diagram of a sampled map and proved that the persistence diagram is determined uniquely and independently of the choice of bases in interval decompositions. However, reconstruction of the homology-induced map depends on the choice of the bases of interval decomposition. Our aim is reconstruction of the underlying maps. Therefore, we must solve the problem of which bases are the best for reconstruction. This problem is anticipated as a subject of our future work. It is expected to be related to the problem of which cycle is optimal for representing the generator of persistent homology (Obayashi 2018).

\section{Compliance with ethical standards}

Conflict of interest The author declares that no conflict of interest exists related to this report or the study it describes.

Open Access This article is licensed under a Creative Commons Attribution 4.0 International License, which permits use, sharing, adaptation, distribution and reproduction in any medium or format, as long as you give appropriate credit to the original author(s) and the source, provide a link to the Creative Commons licence, and indicate if changes were made. The images or other third party material in this article are included in the article's Creative Commons licence, unless indicated otherwise in a credit line to the material. If material is not included in the article's Creative Commons licence and your intended use is not permitted by statutory regulation or exceeds the permitted use, you will need to obtain permission directly from the copyright holder. To view a copy of this licence, visit http://creativecommons.org/licenses/by/4.0/.

\section{References}

Asashiba, H., Escolar, E.G., Hiraoka, Y., Takeuchi, H.: Matrix method for persistence modules on commutative ladders of finite type. Jpn. J. Ind. Appl. Math. 36, 97-130 (2019)

Assem, I., Simson, D., Skowroński, A.: Elements of the Representation Theory of Associative Algebras 1: Techniques of Representation Theory. Cambridge University Press, Cambridge (2006)

Bauer, U., Lesnick, M.: Induced Matchings of Barcodes and the Algebraic Stability of Persistence. In: Proceedings of the Thirtieth Annual Symposium on Computational Geometry (SoCG '14), ACM, New York, NY, USA, pp. 355-364 (2014)

Borsuk, K.: On the imbedding of systems of compacta in simplicial complexes. Fund. Math. 35, 217-234 (1948)

Carlsson, G., de Silva, V.: Zigzag persistence. Found. Comput. Math. 10, 367-405 (2010)

Carlsson, G., Zomorodian, A.: The theory of multidimensional persistence. Discrete Comput. Geom. 42, 71-93 (2009)

Chazal, F., Cohen-Steiner, D., Glisse, M., Guibas, L.J., Oudot, S.Y.: Proximity of Persistence Modules and their Diagrams. In: Proceedings of the Twenty-fifth Annual Symposium on Computational Geometry (SoCG '09), ACM, New York, NY, USA, pp. 237-246 (2009)

Cohen-Steiner, D., Edelsbrunner, H., Harer, J.: Stability of persistence diagrams. Discrete Comput. Geom. 37, 103-120 (2007)

Crawley-Boevey, W.: Decomposition of pointwise finite-dimensional persistence modules. J. Algebra Appl. 14, 1550066 (2015)

Dey, T.K., Fan, F., Wang, Y.: Computing Topological Persistence for Simplicial Maps. In: Proceedings of the 30th Annual Symposium on Computational Geometry (SoCG '14), ACM, New York, NY, USA, pp. 345-354 (2014)

Edelsbrunner, H., Harer, J.: Computational Topology: An Introduction. American Mathematical Society, Providence, Rhode Island (2010) 
Edelsbrunner, H., Jabłoński, G., Mrozek, M.: The persistent homology of a self-map. Found. Comput. Math. 15, 1213-1244 (2015)

Escolar, E.G., Hiraoka, Y.: Computing Optimal Cycles of Homology Groups. In: Nishii, R., Ei, S., Koiso, M., Ochiai, H., Okada, K., Saito, S., Shirai, T. (eds.) A Mathematical Approach to Research Problems of Science and Technology, pp. 101-118. Springer, Tokyo (2014)

Gabriel, P.: Unzerlegbare Darstellungen I. Manuscr. Math. 6, 71-103 (1972)

Harker, S., Kokubu, H., Mischaikow, K., Pilarczyk, P.: Inducing a map on homology from a correspondence. Proc. Am. Math. Soc. 144, 1787-1801 (2016)

Lesnick, M.: The theory of the interleaving distance on multidimensional persistence modules. Found. Comput. Math. 15, 613-650 (2015)

Obayashi, I.: Volume-optimal cycle: tightest representative cycle of a generator in persistent homology. SIAM J. Appl. Algebra Geometry 2(4), 508-534 (2018)

Publisher's Note Springer Nature remains neutral with regard to jurisdictional claims in published maps and institutional affiliations. 